\title{
TanDEM-X: A Satellite Formation for High-Resolution SAR Interferometry
}

\author{
Gerhard Krieger, Member, IEEE, Alberto Moreira, Fellow, IEEE, Hauke Fiedler, Irena Hajnsek, Member, IEEE,
} Marian Werner, Marwan Younis, Member, IEEE, and Manfred Zink

\begin{abstract}
TanDEM-X (TerraSAR-X add-on for Digital Elevation Measurements) is an innovative spaceborne radar interferometer that is based on two TerraSAR-X radar satellites flying in close formation. The primary objective of the TanDEM-X mission is the generation of a consistent global digital elevation model (DEM) with an unprecedented accuracy, which is equaling or surpassing the HRTI-3 specification. Beyond that, TanDEM-X provides a highly reconfigurable platform for the demonstration of new radar imaging techniques and applications. This paper gives a detailed overview of the TanDEM-X mission concept which is based on the systematic combination of several innovative technologies. The key elements are the bistatic data acquisition employing an innovative phase synchronization link, a novel satellite formation flying concept allowing for the collection of bistatic data with short along-track baselines, as well as the use of new interferometric modes for system verification and DEM calibration. The interferometric performance is analyzed in detail, taking into account the peculiarities of the bistatic operation. Based on this analysis, an optimized DEM data acquisition plan is derived which employs the combination of multiple data takes with different baselines. Finally, a collection of instructive examples illustrates the capabilities of TanDEM-X for the development and demonstration of new remote sensing applications.
\end{abstract}

Index Terms-Bistatic SAR, digital elevation model (DEM), formation flying, interferometry, microwave remote sensing, multistatic SAR, synchonization, synthetic aperture radar (SAR).

\section{INTRODUCTION}

D IGITAL elevation models (DEMs) are of fundamental importance for a broad range of commercial and scientific applications [1]-[3]. For example, many geoscience areas, like hydrology, glaciology, forestry, geology, oceanography, and land environment, require precise and up-to-date information about the Earth's surface and its topography. Digital maps are also a prerequisite for reliable navigation, and improvements in their precision need to keep step with the advances in global positioning systems, like GPS and Galileo. In principle, DEMs can be derived from a variety of air- and spaceborne sensors [4], [5]. However, the resulting mosaic of data from different sources with a multitude of horizontal and vertical data, accuracies, formats, map projections, time differences, and resolutions

Manuscript received October 18, 2006; revised February 9, 2007. This work was supported by the German Federal Ministry for Economics and Technology (Förderkennzeichen 50 EE 0601).

The authors are with the Microwaves and Radar Institute, German Aerospace Center (DLR), 82234 Oberpfaffenhofen, Germany (e-mail: Gerhard. Krieger@dlr.de).

Color versions of one or more of the figures in this paper are available online at http://ieeexplore.ieee.org.

Digital Object Identifier 10.1109/TGRS.2007.900693
TABLE I

COMPARISON OF DTED-2 AND HRTI-3 DEM SPECIFICATIONS

\begin{tabular}{|l|c|c|c|}
\hline \multicolumn{1}{|c|}{ Requirement } & Specification & DTED-2 & HRTI-3 \\
\hline Relative Vertical Accuracy & $\begin{array}{c}90 \% \text { linear point-to-point } \\
\text { error over a } 1^{\circ} \times 1^{\circ} \text { cell }\end{array}$ & $\begin{array}{c}12 \mathrm{~m}(\text { slope }<20 \%) \\
15 \mathrm{~m}(\text { slope }>20 \%)\end{array}$ & $\begin{array}{c}2 \mathrm{~m}(\text { slope }<20 \%) \\
4 \mathrm{~m}(\text { slope }>20 \%)\end{array}$ \\
\hline Absolute Vertical Accuracy & $90 \%$ linear error & $18 \mathrm{~m}$ & $10 \mathrm{~m}$ \\
\hline Relative Horizontal Accuracy & $90 \%$ circular error & $15 \mathrm{~m}$ & $3 \mathrm{~m}$ \\
\hline Horizontal Accuracy & $90 \%$ circular error & $23 \mathrm{~m}$ & $10 \mathrm{~m}$ \\
\hline Spatial Resolution & independent pixels & $\begin{array}{c}30 \mathrm{~m} \\
(1 \text { arc sec @ equator })\end{array}$ & $\begin{array}{c}12 \mathrm{~m} \\
(0.4 \operatorname{arc~sec~@~equator~})\end{array}$ \\
\hline
\end{tabular}

is hardly a uniform and reliable data set. The Shuttle Radar Topography Mission (SRTM) [6]-[8] had the challenging goal to meet the requirements for a homogeneous and reliable DEM fulfilling the DTED-2 specification. The coverage of this DEM is, however, principally limited to a latitude range from $56^{\circ} \mathrm{S}$ to $60^{\circ} \mathrm{N}$ due to the inclined orbit of the Space Shuttle and its mapping geometry. Further restrictions apply to the $\mathrm{X}$-band DEM with its wide gaps at lower latitudes and the C-band DEM where the data are available to the public only at an artificially impaired spatial resolution corresponding to the DTED-1 specification. A user survey among a wide range of scientists and potential customers has clearly shown that many applications require both an extended latitudinal coverage and an improved accuracy corresponding to the emerging HRTI-3 standard and comparable to DEMs generated by highresolution airborne radar systems [3], [9]. The acronym HRTI stands for high-resolution terrain information and relates to a DEM specification which uses a fixed latitude and variable longitude grid to represent the elevation data on a global scale [10]. Table I compares the specifications of the DTED-2 and HRTI-3 DEM standards.

The primary objective of the TanDEM-X (TerraSAR-X add-on for Digital Elevation Measurements) mission is the generation of a worldwide, consistent, timely, and highprecision DEM aligned with the HRTI-3 specification as the basis for a wide range of scientific research, as well as for operational and commercial DEM production [2], [9]. This goal will be achieved by means of a second TerraSAR-X-like satellite flying in close orbit configuration with TerraSAR-X. Both satellites will then act as a large single-pass radar interferometer with the opportunity for flexible baseline selection. This enables the acquisition of highly accurate cross- and along-track interferograms without the inherent accuracy limitations imposed by repeat-pass interferometry due to temporal decorrelation and atmospheric disturbances [11]. Besides the primary goal of the mission, several secondary mission objectives based on along-track interferometry (ATI), polarimetric SAR interferometry (PollnSAR), digital 

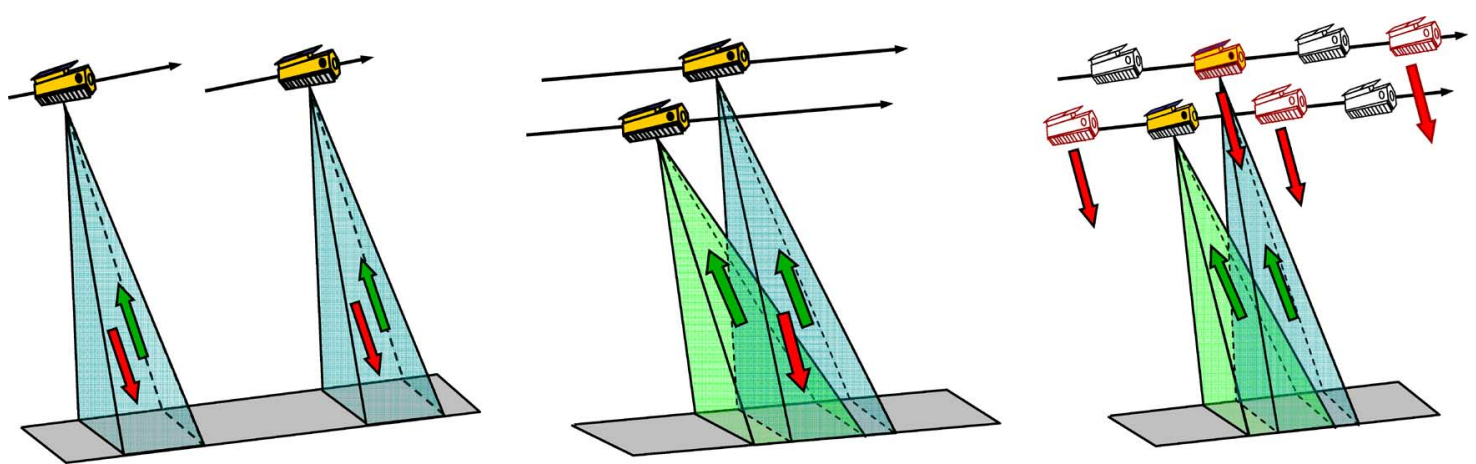

Fig. 1. Examples of data acquisition modes for TanDEM-X. (Left) Pursuit monostatic mode, (middle) bistatic mode, and (right) alternating bistatic mode.

beamforming, and bistatic radar have been defined which represent an important and innovative asset of the mission. TanDEM-X was approved for full implementation by the German government in March 2006 and will be realized in the framework of a public-private partnership (PPP) between the German Aerospace Center (DLR) and EADS Astrium $\mathrm{GmbH}$, as for TerraSAR-X. With a planned launch in summer 2009, TanDEM-X opens a new era in the German space program, providing a major push for the research and development activities and associated techniques and technologies of high-resolution X-band synthetic aperture radar (SAR).

The outline of this paper is as follows. Section II introduces the mission concept including innovative aspects, like orbit selection using the HELIX satellite formation, the employment of multiple interferometric data acquisition modes, and a new synchronization system for bistatic operation which is based on relative phase referencing in X-band via dedicated horn antennas. The achievable DEM accuracy is derived in Section III via a detailed interferometric performance analysis which accounts for the peculiarities of the bistatic SAR data acquisition with multiple baselines. Based on this analysis, we develop in Section IV an optimized data acquisition plan for the derivation of a global DEM in accordance with the HRTI-3 accuracy requirements. Section $\mathrm{V}$ then provides examples for novel radar imaging modes and scientific applications to be demonstrated with TanDEM-X. This paper is concluded in Section VI with a short summary and an outlook on present and future activities.

\section{MISSION CONCEPT}

The TanDEM-X mission is an extension of the TerraSAR-X mission, coflying a second satellite of nearly identical capability in a close formation. The TerraSAR-X satellite (TSX), as basis for TanDEM-X, is not only a high-performance SAR system with respect to SAR image and operational features but it also has already built in all necessary features required for the implementation of the TanDEM-X mission [12]. Examples are additional X-band horn antennas for intersatellite phase synchronization, the availability of a dual-frequency GPS receiver for precise orbit determination, excellent radio-frequency (RF) phase stability of the SAR instrument, and pulse repetition frequency (PRF) synchronization based on GPS as a common time reference. The second satellite (TDX) will be as much as possible a rebuild of TSX with only minor modifications, like an additional cold gas propulsion system for formation fine tuning and an additional S-band receiver to enable a reception of status and GPS position information broadcast by TSX. This guarantees a low development risk, and it offers the possibility for a flexible share of operational functions among the two satellites. ${ }^{1}$ The TDX satellite will be designed for a nominal lifetime of five and one half years and has a nominal overlap with TSX of three years. Note in this context that TSX holds consumables and resources for up to seven years of operation, allowing for a potential prolongation of the TanDEM-X mission duration.

The instruments on both satellites are advanced highresolution X-band SARs based on active phased array technology, which can be operated in Spotlight, Stripmap, and ScanSAR modes with full polarization capability [12]. The center frequency of the instruments is $9.65 \mathrm{GHz}$ with a selectable SAR chirp bandwidth of up to $300 \mathrm{MHz}$. The active phased array antenna, which has an overall aperture size of $4.8 \mathrm{~m} \times 0.7 \mathrm{~m}$, is fixed mounted to the spacecraft body and incorporates 12 panels with 32 waveguide subarrays for both $\mathrm{H}$ and $\mathrm{V}$ polarizations. This enables agile beam pointing and flexible beam shaping.

\section{A. TanDEM-X Operational Modes}

Interferometric data acquisition with the TanDEM-X satellite formation can be achieved in four different operational modes: bistatic, monostatic, alternating bistatic, and simultaneous transmit. Each of these modes will be described in the following. The four interferometric configurations may further be combined with different TSX and TDX SAR imaging modes, like Stripmap, ScanSAR, Spotlight, and Sliding Spotlight.

Operational DEM generation is planned to be performed using the bistatic InSAR stripmap mode shown in Fig. 1 in the middle. This mode uses either TSX or TDX as a transmitter to illuminate a common radar footprint on the Earth's surface. The scattered signal is then recorded by both satellites simultaneously. This simultaneous data acquisition makes dual use of the available transmit power and is mandatory to avoid possible errors from temporal decorrelation and atmospheric disturbances. Prerequisites for bistatic operation are the PRF synchronization and the relative phase referencing between the

\footnotetext{
${ }^{1}$ The current mission scenario employs both satellites, also for monostatic data takes, which is necessary to fulfill the data requirements of the TerraSAR-X mission itself. In this way, the TanDEM-X mission goals can be achieved without jeopardizing the TerraSAR acquisitions.
} 

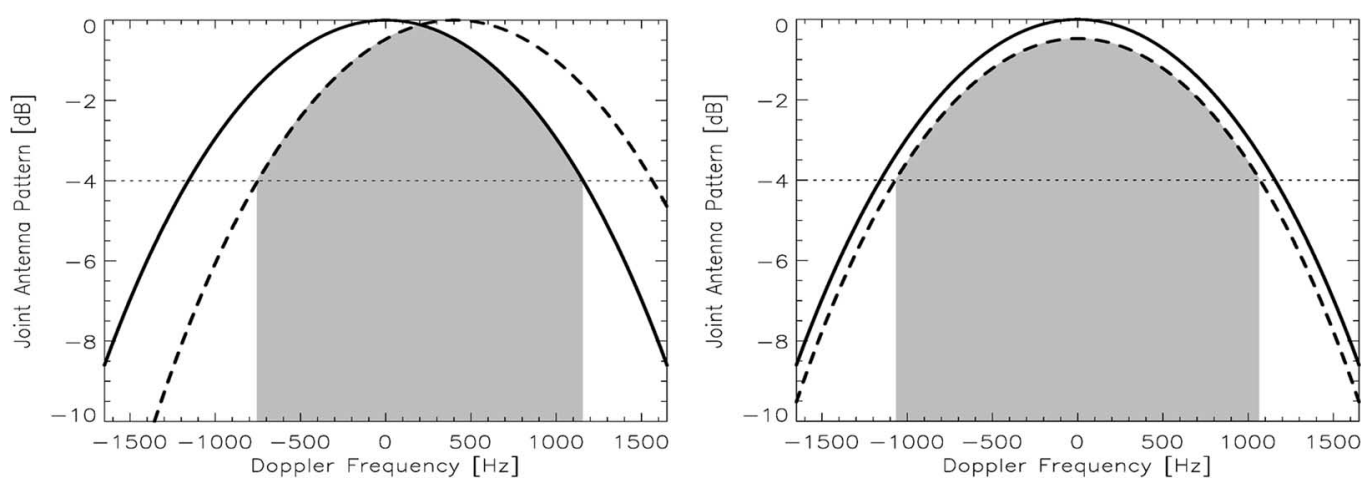

Fig. 2. Azimuth weighting of Doppler spectra for beamsteering to a (left) joint azimuth footprint and with (right) independent zero Doppler steering. The alongtrack displacement is $1 \mathrm{~km}$, and the slant range is $600 \mathrm{~km}$. Solid and dashed lines show weightings of mono- and bistatic Doppler spectra, respectively. The gray areas indicate the common Doppler spectra which are, in this example, confined by a maximum gain loss of $4 \mathrm{~dB}$, as shown by the dotted lines.

two satellites. These challenging topics will be discussed in more depth in Section II-B and C.

Another essential requirement for radar interferometry on natural surfaces is a sufficient overlap of the two recorded Doppler spectra. For this, we note first that the instantaneous Doppler shift between the monostatic and the bistatic SAR acquisitions is given by [13]

$$
\begin{aligned}
\Delta f_{\text {Dop }} & =\frac{1}{\lambda}\left[2 \cdot \frac{\partial r_{1}}{\partial t}-\left(\frac{\partial r_{1}}{\partial t}+\frac{\partial r_{2}}{\partial t}\right)\right] \\
& =\frac{1}{\lambda}\left(\frac{\left(\vec{\nu}_{1}-\vec{\nu}_{\mathrm{E}}\right) \circ \vec{p}_{1}}{\left\|\vec{p}_{1}\right\|}-\frac{\left(\vec{\nu}_{2}-\vec{\nu}_{\mathrm{E}}\right) \circ \vec{p}_{2}}{\left\|\vec{p}_{2}\right\|}\right)
\end{aligned}
$$

where $\lambda$ denotes the wavelength, $r_{1}$ and $r_{2}$ are the ranges from the two satellites to a given point on the ground, o denotes the scalar product, $\vec{\nu}_{1}$ and $\vec{\nu}_{2}$ are the velocity vectors of the two satellites in an inertial reference frame, $\vec{p}_{1}$ and $\vec{p}_{2}$ are the instantaneous look vectors which connect the actual satellite positions with the point on the ground, and $\vec{\nu}_{\mathrm{E}}$ is the velocity vector of the scatterer due to Earth rotation. For the following analysis, we assume identical velocity vectors for the two satellites, since their magnitude difference will be less than $1 \mathrm{~m} / \mathrm{s}$ (for $a \cdot \Delta e<500 \mathrm{~m}$, cf., Section II-D) and their angular difference stays always below $0.001^{\circ}$. We assume furthermore that the illuminator satellite performs total zero Doppler steering to compensate the additional Doppler shift from Earth rotation with regard to the monostatic SAR image [14]. The corresponding yaw and pitch angles are smaller than $4^{\circ}$ and $0.1^{\circ}$ (cf., [12]), respectively, and their additional impact on the Doppler difference can well be neglected in the following. Equation (1) may then be approximated by

$$
\Delta f_{\text {Dop }} \approx \frac{\nu \cdot d_{\text {along }}}{\lambda \cdot r}
$$

where $\nu$ is the satellite velocity, $d_{\text {along }}$ is the along-track displacement between the satellites, and $r$ is the mean slant range $r=\left(r_{1}+r_{2}\right) / 2$. An along-track displacement of $1 \mathrm{~km}$ will hence result in an instantaneous Doppler shift of $\sim 410 \mathrm{~Hz}$ for a slant range of $600 \mathrm{~km}$ which corresponds in TanDEM-X to an incident angle of $33^{\circ}$.
We may now compare two different solutions for the acquisition of the bistatic SAR image. The first alternative steers the antenna beam of the second satellite such that the footprints of both antennas yield an almost perfect overlap on the ground. The relative distortion of the two antenna footprints can be neglected due to the small satellite displacements considered for bistatic cross-track interferometry. The recorded Doppler spectra are then confined by the same joint antenna pattern, and we obtain from (2) a mutual displacement between the monostatic and the bistatic Doppler centroids which varies between 330 and $420 \mathrm{~Hz}$ in case of a 1-km along-track displacement and the incident angle range considered in Section III-A. This corresponds to $\sim 20 \%$ of the processed azimuth bandwidth, thereby reducing the number of independent looks for interferometric phase estimation as outlined in Section III-B. The mutual shift between the two Doppler spectra can be avoided by employing the zero Doppler steering independently for both satellites. The weighting of the bistatic SAR spectrum by the joint azimuth antenna pattern can then be approximated by

$$
\begin{aligned}
& W_{\text {bistat }}\left(f_{\text {Dop }} ; \Delta f_{\text {Dop }}\right)=\operatorname{sinc} {\left[\frac{\pi l}{2 \nu}\left(f_{\text {Dop }}-\Delta f_{\text {Dop }}\right)\right] } \\
& \cdot \operatorname{sinc}\left[\frac{\pi l}{2 \nu}\left(f_{\text {Dop }}+\Delta f_{\text {Dop }}\right)\right]
\end{aligned}
$$

where $l$ is the antenna length, and $\operatorname{sinc}(x)=\sin (x) / x$. Fig. 2 compares the azimuth weightings of the mono- and bistatic Doppler spectra for the two beamsteering alternatives. The second alternative yields, in general, an improved spectral overlap on the cost of a reduced maximum gain for the bistatic SAR image. For TanDEM-X, this gain loss is less than $0.5 \mathrm{~dB}$ for the along-track displacements below $1 \mathrm{~km}$. A mispointing of the two antenna beams may further reduce the gain. By noting that the pointing accuracy in TerraSAR-X is $0.01^{\circ}$, which corresponds to $3 \%$ of the $3-\mathrm{dB}$ azimuth beamwidth, we obtain in the worst case of opposite pointing errors an additional gain loss of $0.1 \mathrm{~dB}$, which has an almost negligible effect on the overall interferometric performance. Based on this and further analyses in Section III, it was decided that TanDEM-X employs the second alternative of independent zero Doppler steering during the DEM data acquisition phase. This has the operational 

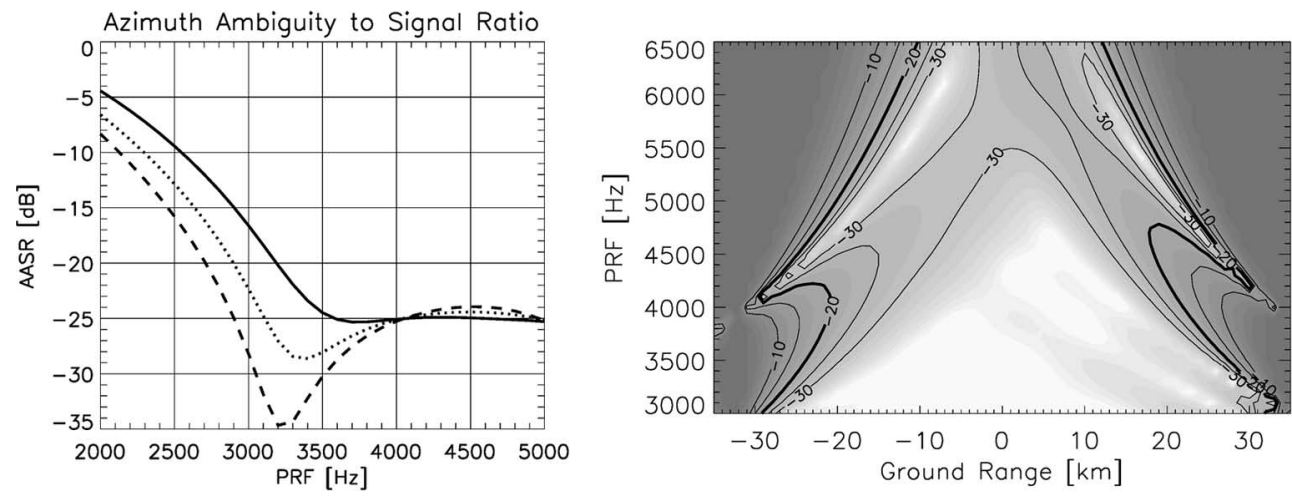

Fig. 3. (Left) Azimuth ambiguities as a function of the PRF for processed bandwidths of (solid) 2266, (dotted) 1600, and (dashed) 1066 Hz. (Right) Range ambiguities as a function of ground range position and PRF for an incident angle of $35^{\circ}$.

advantage that the steering angles become independent from the actual along-track separation between the two satellites.

A secondary DEM generation mode is the pursuit monostatic InSAR mode which is shown in Fig. 1 on the left. In this mode, the two satellites are operated independently from each other, thereby avoiding the need for synchronization. The along-track distance should be larger than $10 \mathrm{~km}$ to avoid RF interference between the radar signals. Temporal decorrelation is still small for most terrain types except vegetation at moderate to high wind speeds as well as for water. The interferometric height sensitivity is doubled with respect to the bistatic operation, which means that the baseline determination has to be more accurate by the same factor. Neither pulse nor phase synchronization is required in the pursuit monostatic mode. This mode is hence well suited as a backup solution in the case of synchronization problems and/or problems with close formation flying. The selection of appropriate orbits for the pursuit monostatic mode has also to take into account the rotation of the Earth, which leads - if uncompensated - to a latitude-dependent additional cross-track baseline between the two monostatic data acquisitions. Hence, a relative shift between the right ascensions of the ascending nodes of the two satellite orbits will be required if one wants to achieve the same baselines as in the bistatic mode employing a close formation. Vice versa, one may exploit an adaptation of the along-track separation to adjust the cross-track baseline without a fuel-consuming shift of the right ascension of the ascending node. As explained later on, this may, e.g., be used to acquire interferometric data in a crossing orbit configuration without the necessity to change the radar operation from a right- to a left-looking mode. Monostatic data takes are planned during the commissioning phase, in an intermediate phase when the satellites are separated from each other for a formation swap, and at the end of the mission when the satellite formation is flown with an increasing along-track separation.

A third operational mode is the alternating bistatic mode, where the transmitter is switched on a pulse-to-pulse basis. The scattered signal from the ground is then recorded by both receivers simultaneously, as shown in Fig. 1 on the right. The alternating bistatic mode acquires two monostatic and two bistatic SAR images during a single pass of the satellite formation. For an ideal system and under the assumption of scatterer reciprocity, the two bistatic SAR images would become equal.
Systematic deviations between the two bistatic SAR images will be mainly due to differences in the oscillator frequencies of the two tandem satellites, while deviations from reciprocity can well be neglected for the intended interferometric acquisitions due to the small bistatic angle which will be below $0.1^{\circ}$. A comparison of the two bistatic images is hence well suited for the measurement of oscillator-induced phase errors, thereby enabling an accurate phase calibration of the bistatic SAR interferometer. After phase calibration, the two bistatic images can be combined into a single bistatic SAR image with double PRF. For the cross-track interferometry, it is now possible to form two interferograms with different phase-toheight sensitivities.

1) The combination of one monostatic image and a bistatic image yields a cross-track interferogram with a height of ambiguity of $h_{\mathrm{amb}}=\left(\lambda r \sin \left(\theta_{\mathrm{i}}\right)\right) / B_{\perp}$, where $\lambda$ is the wavelength, $r$ is the slant range, $\theta_{\mathrm{i}}$ is the incident angle, and $B_{\perp}$ is the baseline perpendicular to the line of sight. Either the first or the second monostatic image can be selected, and a combination of both interferograms can be used to improve both the phase calibration and the phase stability.

2) The combination of the two monostatic SAR images yields a second interferogram with a double phase-toheight sensitivity, resulting in a height of ambiguity of $h_{\mathrm{amb}}=\left(\lambda r \sin \left(\theta_{\mathrm{i}}\right)\right) /\left(2 B_{\perp}\right)$.

The use of alternating transmitters in the bistatic mode allows for the simultaneous acquisition of two cross-track interferograms with phase-to-height sensitivities differing by a factor of two. This will facilitate the process of interferometric phase unwrapping since it is now possible to reduce the interferometric baseline without a loss in height accuracy if compared to the bistatic mode. The simultaneous availability of mono- and bistatic SAR interferograms will provide additional information in case of volume scattering [15], and it may help to distinguish between direct and double-bounce scattering from the ground [16].

The major drawback of the alternating bistatic mode is an increased susceptibility of the two monostatic SAR images to range and azimuth ambiguities. This can be seen in Fig. 3 which shows the integrated ambiguity-to-signal ratios (ASRs) for TanDEM-X. The figure on the left reveals that the minimum 


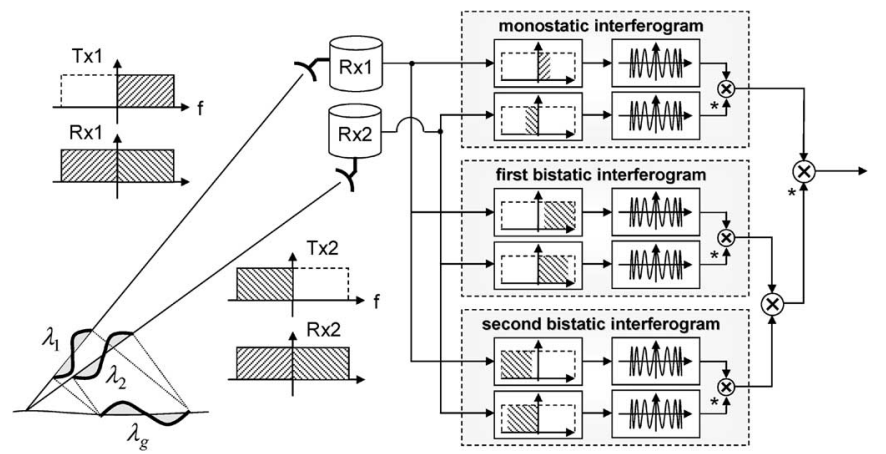

Fig. 4. (Left) Illustration of simultaneous transmit mode and (right) derivation of oscillator phase errors by comparing the phase of the monostatic and bistatic interferograms.

PRF should be on the order of $3 \mathrm{kHz}$ for each channel to ensure a sufficiently low azimuth ASR (AASR). This implies that the minimum PRF for both channels is on the order $6 \mathrm{kHz}$, which will, in turn, reduce the unambiguous swath width to $\sim 25 \mathrm{~km}$ for an incident angle of $35^{\circ}$, as shown in the right-hand side of Fig. 3. Note that this reduction quickly increases with higher incident angles. We will employ the alternating bistatic mode mainly for system verification, calibration, and demonstration of new applications, which are based on the joint evaluation of mono- and bistatic SAR images and interferograms.

The drawbacks of the alternating bistatic mode can be avoided by a fourth operational mode, where the two spacecrafts transmit their radar pulses at the same time [17]. To prevent the RF interferences, the signal spectrum will be split into two disjoint frequency bands, and each satellite illuminates the scene with one of these subbands, as shown in Fig. 4 on the left. The full spectrum of the scattered signal is then recorded by both receivers simultaneously. This enables not only an efficient share of the transmit energy between the two spacecrafts but it also allows for the unambiguous acquisition of two monostatic and two bistatic SAR images in a single pass. From these images, one may then form two bistatic interferograms, one for each subband. In addition, a low-resolution monostatic interferogram can be obtained by exploiting the wavenumber shift [18], which allows for coherent interferometric data acquisitions even in case of nonoverlapping RF frequency bands as long as the satellite orbits are separated in the crosstrack direction. By comparing now the redundant information between the low-resolution monostatic interferogram and its bistatic counterparts as shown in Fig. 4 on the right, one may extract low-frequency phase errors resulting from mutual drifts of the oscillator phases in the two spacecrafts. The phase in the two bistatic interferograms can be approximated by [19]

$\Delta \varphi_{\text {bistat }}(t)=2 \pi \cdot m \cdot \Delta f_{\text {osc }} \cdot t+m \cdot \Delta \varphi_{\text {osc }}(t)+\varphi_{\text {topo }}$

where $m$ is the frequency up-conversion factor, $\Delta f_{\text {osc }}=$ $f_{\text {osc }, 1}-f_{\text {osc }, 2}$ is a constant frequency offset between the two local oscillators, $\Delta \varphi_{\mathrm{osc}}(t)=\varphi_{\mathrm{osc}, 1}(t)-\varphi_{\mathrm{osc}, 2}(t)$ is the timevarying phase difference due to the mutually uncorrelated phase noise from each local oscillator, and $\varphi_{\text {topo }}$ is the desired topographic phase. By combining the two bistatic interferograms in a fourth-order interferogram and demodulating it with the phase
TABLE II

TANDEM-X SYSTEM PARAMETERS

\begin{tabular}{|l|c|l|c|}
\hline Parameter & Value & Parameter & Value \\
\hline Satellite Height (equator) & $511.5 \mathrm{~km}$ & Antenna Length & $4.8 \mathrm{~m}$ \\
\hline Carrier Frequency & $9.65 \mathrm{GHz}$ & Antenna Width & $0.7 \mathrm{~m}$ \\
\hline Chirp Bandwidth & $100 \mathrm{MHz}$ & Antenna T/R Elements & $32 \times 12$ \\
\hline Sampling Frequency & $110 \mathrm{MHz}$ & Antenna Tapering & linear phase \\
\hline Mutual Swath Overlap & $\geq 4 \mathrm{~km}$ & Antenna Mounting & $33.8^{\circ}$ \\
\hline Peak Tx Power & $2260 \mathrm{~W}$ & Quantization & 3 bits $/$ sample \\
\hline Duty Cycle & $18 \%$ & Image Misregistration & $<0.1$ pixel \\
\hline Noise Figure T/R Module & $4.3 \mathrm{~dB}$ & Along-Track Baseline & $<1 \mathrm{~km}$ \\
\hline Losses (atmosphere, radiator,...$)$ & $3.1 \mathrm{~dB}$ & Sigma Nought Model $(90 \%,[30])$ & Soil \& Rock, VV \\
\hline Azimuth Processing Losses & $<1.5 \mathrm{~dB}$ & Indep. Post Spacing & $12 \mathrm{~m} \times 12 \mathrm{~m}$ \\
\hline
\end{tabular}

from the monostatic interferogram, as shown in Fig. 4 on the right, one obtains a direct measure for the bistatic phase error

$$
\Delta \varphi_{\mathrm{error}}(t)=4 \pi \cdot m \cdot \Delta f_{\mathrm{osc}} \cdot t+2 \cdot m \cdot \Delta \varphi_{\mathrm{osc}}(t)
$$

which can be used to correct the phase error in the two bistatic SAR interferograms. Note that the topographic phase $\varphi_{\text {topo }}$ is canceled, since the combination of both bistatic interferograms leads to the same phase-to-height sensitivity as in the monostatic interferogram.

The phase error in (5) typically represents a low-frequency signal with a narrow power spectrum and, thereby, affects almost exclusively the evolution of the azimuth signal while preserving the phase of each range line up to a constant offset. One may combine all range and multiple azimuth samples to identify mutual phase drifts with high accuracy notwithstanding the low common bandwidth in the monostatic interferogram. For a first rough estimate of the expected performance, we note that the spectral shift between the two monostatic SAR acquisitions is given by [20]

$$
\Delta f=\frac{f_{\mathrm{c}} \cdot B_{\perp}}{r \cdot \tan \left(\theta_{\mathrm{i}}\right)}
$$

where $f_{\mathrm{c}}$ is the radar center frequency. An effective cross-track baseline of $300 \mathrm{~m}$ will lead with the TanDEM-X parameters of Table II to the spectral shifts of 8.6 and $4.1 \mathrm{MHz}$ for incident angles of $30^{\circ}$ and $45^{\circ}$, respectively. The phase errors for a single range line can then be approximated by

$$
\begin{aligned}
\sigma_{\varphi} & \approx \frac{1}{\sqrt{T_{\mathrm{avg}} \cdot \Delta f \cdot \mathrm{SNR}}} \\
& \approx \sqrt{\frac{\lambda \cdot r}{2 \cdot \Delta s \cdot B_{\perp} \cdot \cos \left(\theta_{\mathrm{i}}\right) \cdot \mathrm{SNR}}}
\end{aligned}
$$

which yields for a swath width of $\Delta s=30 \mathrm{~km}$, a signal-tonoise ratio (SNR) of $10 \mathrm{~dB}$, and the incident angles of $30^{\circ}$ and $45^{\circ}$ phase errors of $0.6^{\circ}$ and $0.75^{\circ}$, respectively. A lower SNR and additional coherence losses will reduce this accuracy, but this could be compensated by extending the averaging period $T_{\text {avg }}$ over not only one but multiple range lines. The simultaneous transmit mode with split frequency bands enables an accurate phase synchronization without the susceptibility to ambiguities if compared to the alternating transmit mode. The price to be paid is a lower range resolution for each individual SAR image and a loss of coherence between the monostatic 

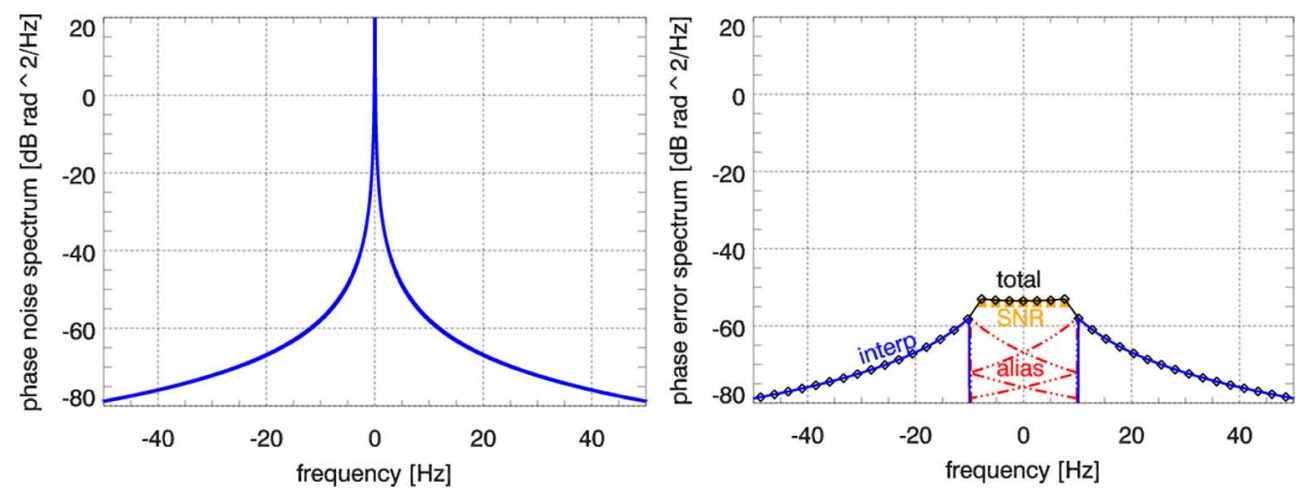

Fig. 5. (Left) X-band phase noise spectrum $S_{\varphi}(f)$ versus frequency offset from the carrier. (Right) Remaining phase error contributions $E_{\text {interpol }}(f)$, $E_{\text {alias }}(f)$, and $E_{\mathrm{SNR}}(f)$ after periodic synchronization with $f_{\mathrm{syn}}=20 \mathrm{~Hz}$ for an SNR of $35 \mathrm{~dB}$.

images for a significant part of the frequency spectrum. In case of high bandwidth interferometric systems like TanDEM-X, which allow for multiple looks in range, the resolution loss in the bistatic interferogram is more than compensated by the higher SNR in each subband. TanDEM-X will employ this new mode for system calibration and verification, the demonstration of novel SAR techniques and applications, as well as a backup solution in case of problems with the phase synchronization link to be introduced in the next section.

\section{B. Relative Phase Referencing}

A peculiarity of the standard bistatic data acquisition mode is the use of independent oscillators for modulation and demodulation of the radar pulses. Any deviation between the two oscillators will cause a residual modulation of the recorded azimuth signal. The impact of oscillator phase noise in the bistatic SAR has been analyzed in [19], where it is shown that oscillator noise may cause significant errors in both the interferometric phase and the SAR focusing. The stringent requirements for interferometric phase stability will require relative phase referencing between the two SAR instruments. For this purpose, both TSX and TDX are equipped with six dedicated synchronization horn antennas covering the full solid angle for a mutual exchange of radar pulses between the two satellites [21]. The nominal bistatic SAR data acquisition is periodically interrupted, and a radar pulse is redirected from the main SAR antenna to one of these six horn antennas pointing in the direction of the second spacecraft. The pulse is then recorded by the corresponding synchronization horn antenna on the other satellite which transmits back a short synchronization pulse. In this way, the mutual phase referencing becomes independent of the actual distance between the satellites. The synchronization pulses are then processed on the ground to extract a correction phase which compensates the oscillator phase error in the bistatic SAR interferogram.

In order to characterize the accuracy of the synchronization process, we note first that the phase errors are conveniently described in the frequency domain by a phase noise power spectral density function $S_{\varphi}(f)$ [22]. The left-hand side of Fig. 5 shows for $\operatorname{TSX} S_{\varphi}(f)$ that is up-converted to the $\mathrm{X}$-band as a function of the frequency offset from the carrier. The accuracy of the synchronization process is then determined by how accurately the oscillator phase difference matches the phase derived from the synchronization pulses. The error sources influencing the quality of the compensation phase can be grouped into two main categories: 1) errors due to the finite synchronization frequency $f_{\text {syn }}$ and 2) errors due to the finite SNR of the synchronization link. The main contributions from the first category are interpolation errors

$$
E_{\text {interpol }}(f)=S_{\varphi}(f) \quad \text { for } \quad|f| \geq f_{\text {syn }}
$$

and aliasing errors

$$
\begin{array}{r}
E_{\text {alias }}(f)=\sum_{\substack{i \neq 0 \\
i=-\infty}}^{i=\infty} S_{\varphi}\left(f+i \cdot f_{\mathrm{syn}}\right) \cdot\left|H_{\mathrm{LP}}\left(f+i \cdot f_{\mathrm{syn}}\right)\right|^{2} \\
\text { for }|f|<f_{\mathrm{syn}}
\end{array}
$$

which are both due to the low synchronization frequency $f_{\text {syn }}$ if compared to the bandwidth of the phase noise spectrum $S_{\varphi}(f)$ [23]. The weighting function $\left|H_{\mathrm{LP}}(f)\right|=\cos \left(\pi f \tau_{\text {sys }}\right)$ in (9) accounts for the alternating synchronization scheme, leading to a short time delay $\tau_{\text {sys }}$ between the two transmit events. The right-hand side of Fig. 5 shows the power spectral densities $E_{\text {interpol }}(f)$ and $E_{\text {alias }}(f)$.

The second category of phase errors is caused by the receiver noise which is well characterized by a white spectrum within the receiver bandwidth. Sampling will cause all noise spectral contributions to be folded into the frequency interval $|f|<f_{\text {syn }}$ and, thus, does not lead to noise reduction. The error spectrum is hence given by

$$
E_{\mathrm{SNR}}(f)=\frac{1}{4 \mathrm{SNR} \cdot f_{\mathrm{syn}}} \text { for } \quad|f|<f_{\mathrm{syn}} .
$$

The resulting phase errors are then derived by multiplying their spectra with the azimuth compression filter and integrating the result over frequency. Here, the azimuth compression filter is a lowpass which accounts for temporal averaging in accordance with the processed Doppler bandwidth. The total error budget is finally given by

$$
\sigma_{\text {link }}^{2}=\sigma_{\text {interp }}^{2}+\sigma_{\text {alias }}^{2}+\frac{1}{2} \sigma_{\mathrm{SNR}}^{2}
$$




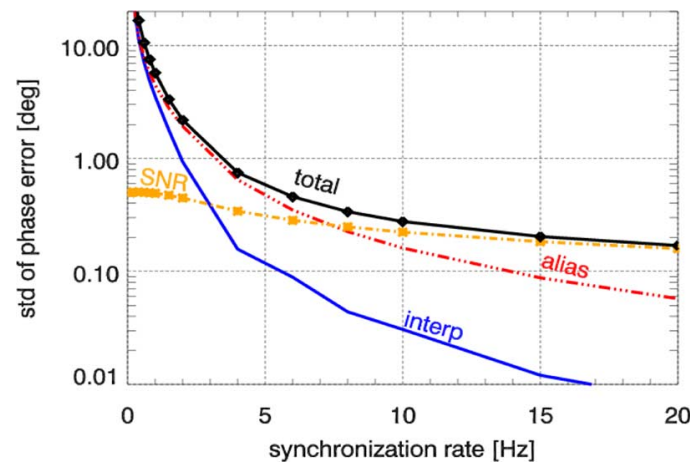

Fig. 6. Standard deviation of the synchronization link phase error contributions as a function of the synchronization frequency. The SNR and the azimuth integration time are $35 \mathrm{~dB}$ and $0.5 \mathrm{~s}$, respectively.

where $\sigma_{\text {interp }}^{2}$ and $\sigma_{\text {alias }}^{2}$ are the interpolation and aliasing error contributions, respectively, and $\sigma_{\mathrm{SNR}}^{2} / 2$ is the receiver noise from the bidirectional synchronization link. Fig. 6 shows the predicted phase error contributions as a function of the synchronization frequency. The SNR is $35 \mathrm{~dB}$, and the azimuth integration time is $0.5 \mathrm{~s}$ in this example. For synchronization frequencies above $10 \mathrm{~Hz}$, the phase error is dominated by the receiver noise. The actual SNR varies with the distance between the satellites as well as their relative attitude. For the typical DEM data acquisition mode with baselines below $1 \mathrm{~km}$, the SNR will be on the order of 30 to $40 \mathrm{~dB}$, and a phase error below $1^{\circ}$ can be achieved for synchronization frequencies above $5 \mathrm{~Hz}$.

\section{Relative Time Referencing}

TSX and TDX trigger the start of a data take via GPS, but the PRF timing is then internally derived from the ultrastable oscillators (USOs). A deviation of the two USO frequencies will lead to a drift of the receiving window of one satellite with respect to the transmit event of the other satellite and may, in this way, prevent a proper recording of the echo signal. TanDEM-X accounts for this by introducing leap pulse repetition intervals (PRIs) which readjust the position of the receiving window. In order to determine the interval and the duration of these leap PRIs, it is necessary to predict the time difference between the two satellites. Since the data take start time is derived from the GPS to an accuracy of 1-2 $\mu$ s, only a relative time drift after the start of the data take has to be considered. The time difference between the two satellites is then given by

$$
\Delta t_{\mathrm{sat}}(t)=\frac{\Delta f_{1}-\Delta f_{2}}{f_{\mathrm{USO}}} \cdot t
$$

where $\Delta f_{1}$ and $\Delta f_{2}$ denote the deviations from the nominal USO frequency $f_{\text {USO }}$. A frequency difference $\Delta f_{1}-\Delta f_{2}$ of, e.g., $60 \mathrm{~Hz}$ between the two $60-\mathrm{MHz}$ oscillators would cause a relative drift of the receiving window of $1.0 \mu \mathrm{s} / \mathrm{s}$ and requires therefore a leap PRI every second in order to keep the shift of the receiving window below $1 \mu \mathrm{s}$. The commanding of appropriate leap PRIs requires the frequency difference $\Delta f_{1}-\Delta f_{2}$ to be known a priori. A very accurate measurement of the actual frequency difference can, for example, be derived from an evaluation of the synchronization pulses (cf. Section II-B). However, the frequency difference changes due to the aging of the two USOs. By allowing an additional time error of $\Delta t_{\text {sat } \max }=5 \mu$ s at the end of a 600-s data take, the value of $\Delta f_{1}-\Delta f_{2}$ needs to be known with an accuracy of $0.5 \mathrm{~Hz}$ at the time of the data take. Considering the aging of the TerraSAR-X USO, the values of $\Delta f_{1,2}$ may change by $6 \mathrm{~Hz} /$ year. This means that the frequency difference $\Delta f_{1}-\Delta f_{2}$ should be actualized every two weeks.

\section{Orbit Configuration and Formation Flying}

The TanDEM-X operational scenario requires a coordinated operation of two satellites flying in close formation. The adjustment parameters for the formation are the node line angle, the angle between the perigees, the orbit eccentricities, and the phasing between the satellites. With these parameters, several options have been investigated, and the HELIX satellite formation shown in Fig. 7 has finally been selected for the operational DEM generation. This formation combines an out-of-plane (horizontal) orbital displacement by different ascending nodes with a radial (vertical) separation by different eccentricity vectors resulting in a helixlike relative movement of the satellites along the orbit [24]. Since crossing of the satellite orbits does not exist, one may now allow for arbitrary shifts of the satellites along their orbits. This enables a safe spacecraft operation without the necessity for autonomous control [25]. It is furthermore possible to optimize the along-track displacement at predefined latitudes for different applications: cross-track interferometric applications shall use along-track baselines which are as short as possible, to ensure an optimum overlap of the Doppler spectra and to avoid temporal decorrelation in vegetated areas, while other applications, like ATI or superresolution, require selectable along-track baselines in the range from one hundred meters up to several kilometers.

The HELIX formation enables an interferometric mapping of the complete Earth surface with a stable height of ambiguity using a small number of formation settings which will be derived in Section IV. Southern and northern latitudes can be mapped with the same formation by using ascending orbits for one and descending orbits for the other hemisphere, as shown in Fig. 7 on the right.

For a more detailed treatment of the peculiarities of the HELIX formation, we note first that, due to the nonspherical geopotential of the Earth, a satellite is exposed to nonlinear forces, and its orbit will possess long periodic variations. If these long periodic variations vanish, the satellite's orbit is defined as frozen. The main parameter, which describes such an orbit, is the so-called frozen eccentricity, which can be approximated by [27]

$$
\begin{aligned}
e_{\mathrm{F}}=\mid-\frac{1}{2} \frac{J_{3}}{J_{2}} \frac{R_{\mathrm{E}}}{a} \sin (i)-\frac{5}{8} \frac{J_{5}}{J_{2}} \frac{R_{\mathrm{E}}^{3}}{a^{3}} \sin (i) \\
\cdot\left[1-9 \cos ^{2}(i)-\frac{24 \cos ^{4}(i)}{1-5 \cos ^{2}(i)}\right] \mid
\end{aligned}
$$



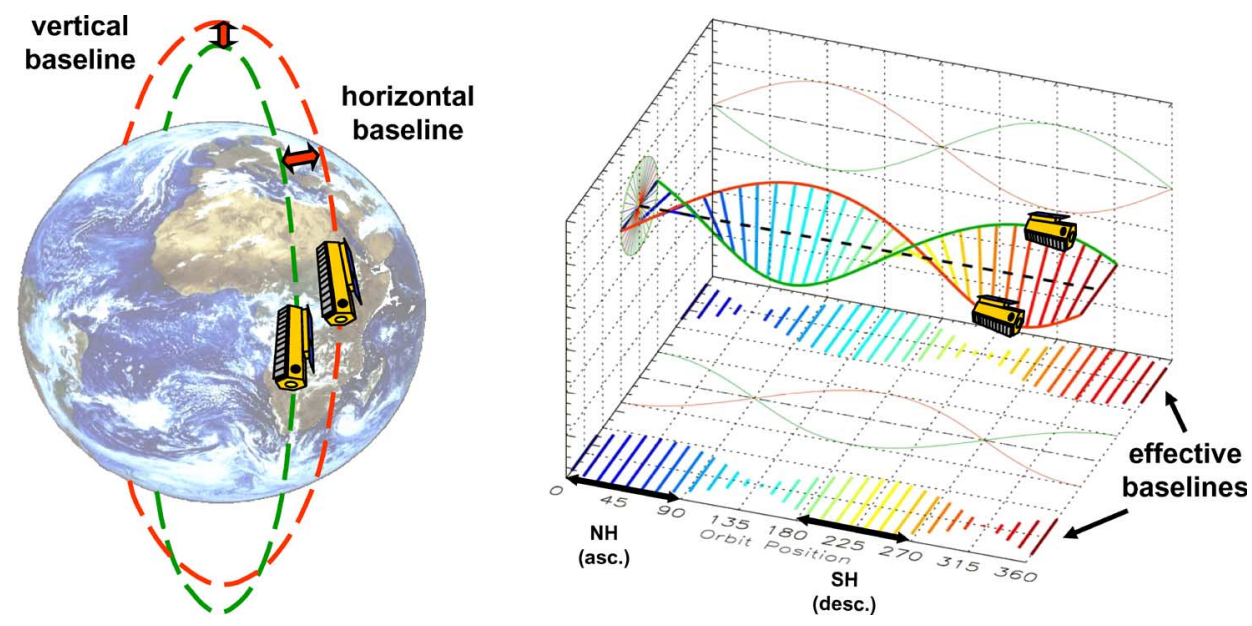

Fig. 7. HELIX satellite formation for TanDEM-X. (Left) Orbital arrangement. (Right) Cross-track baselines as function of the orbit position. The positions correspond to one complete orbit cycle, where NH and SH mean Northern and Southern Hemispheres, respectively.

taking into account the zonal coefficients up to $J_{5}$. Here, $R_{\mathrm{E}}$ denotes the Earth's radius, $a$ is the mean semimajor axis, and $i$ is the mean inclination. By definition, the mean argument of perigee is $90^{\circ}$. If the eccentricity deviates from $e_{\mathrm{F}}$, the satellite orbit is no longer dimensionally stable, and its perigee will start moving around the orbit. This motion is called libration, and its period $T_{\text {libration }}$ is given by

$$
T_{\text {libration }}=\left|\frac{2 \pi}{86400 \cdot \dot{\omega}}\right|
$$

where $\dot{\omega}$ is the short periodic variation of the argument of perigee which can, for small eccentricities, be approximated by [27]

$$
\dot{\omega} \approx-\frac{3}{4} \sqrt{\mu_{\text {Earth }}} J_{2} R_{\mathrm{E}}^{2} a^{-\frac{7}{2}} \cdot\left(1-5 \cos ^{2}(i)\right)
$$

where $\mu_{\text {Earth }}=3.98 \cdot 10^{14} \mathrm{~m}^{3} / \mathrm{s}^{2}$ is the gravitational coefficient of the Earth. Since the TSX's eccentricity is set to frozen, TDX cannot orbit with a frozen eccentricity, and it will perform the motion of libration over a period of $T_{\text {libration }} \approx 104$ days.

To describe the relative motion between the two satellites, a Cartesian corotating coordinate system is built up [28]: the $x$-axis (along-track separation) points into flight direction, the $y$-axis (horizontal cross-track separation) is parallel to the orbit momentum vector, and the $z$-axis (radial separation) points from the Earth's center of mass to the satellite's center of mass. Mathematically, the unit vectors may be expressed by

$$
\begin{aligned}
\vec{e}_{\text {along }} & =\vec{e}_{\text {cross }} \times \vec{e}_{\text {radial }} \\
\vec{e}_{\text {cross }} & =\vec{s} \times \vec{\nu} /\|\vec{s} \times \vec{\nu}\| \\
\vec{e}_{\text {radial }} & =\vec{s} / s
\end{aligned}
$$

where $\vec{\nu}$ is the velocity vector of the satellite, and $\vec{s}$ is the position vector of the satellite. The relative motion between TDX and TSX is then given by

$$
\begin{aligned}
\vec{s}_{\mathrm{TDX}}-\vec{s}_{\mathrm{TSX}}=\Delta r_{\text {along }} & \cdot \vec{e}_{\text {along }}+\Delta r_{\text {cross }} \\
\cdot & \vec{e}_{\text {cross }}+\Delta r_{\text {radial }} \cdot \vec{e}_{\text {radial }} .
\end{aligned}
$$

In case of near-circular orbits in close formation, the motion may be linearized [29], and the components of the relative position vector $\vec{s}_{\text {TDX }}-\vec{s}_{\text {TSX }}$ are then given by

$$
\begin{aligned}
\Delta r_{\text {along }} & =-2 a \Delta e \cos (u+\psi)+\Delta x_{\text {along }} \\
\Delta r_{\text {cross }} & =-a \Delta i \cos (u) \\
\Delta r_{\text {radial }} & =-a \Delta e \sin (u+\psi)
\end{aligned}
$$

where $\Delta e$ is the difference between the two eccentricity vectors, $u$ is the argument of latitude, $\psi$ is the libration angle defined as the difference between the eccentricity and the inclination vector [26], and $\Delta x_{\text {along }}$ is an arbitrary alongtrack shift. Because both satellites orbit at the same inclination, the difference in $\Delta i$ is set up by different ascending nodes only. Therefore, a helix may be defined by three parameters: the vertical separation $a \cdot \Delta e$, the horizontal separation $a \cdot \Delta i$, and the libration angle $\psi$. Note that $\psi$ varies between $0^{\circ}$ and $360^{\circ}$ during the libration period $T_{\text {libration. }}$. A stable acquisition geometry requires a constant libration phase $\psi$. This is achieved via daily orbit maneuvers using the cold gas propulsion system on TDX which necessitate a $\Delta \nu$ of approximately $3.3 \cdot 10^{-05}$. $(a \cdot \Delta e) / \mathrm{s}$ per day.

For libration phases of $\psi=0^{\circ}$ and $\psi=180^{\circ}$, the two orbits are spatially separated with no collision risk, while for $\psi=90^{\circ}$ and $\psi=270^{\circ}$, the satellites are separated at the northern/ southern turns only in along track, leaving a high collision risk. If the satellites are controlled at an intermediate libration phase of, e.g., $\psi=210^{\circ}$, the time to reach $\psi=270^{\circ}$ will be $\sim 17$ days, which is enough time to react in case that one satellite goes into safe mode or has problems on the command link. The motion of libration can even be used for a fine tuning of the cross-track baselines by adapting the frequency of the orbit maneuvers.

\section{Performance Analysis}

This section investigates the interferometric performance of TanDEM-X, assuming an interferometric data acquisition in bistatic stripmap mode. Table II summarizes the main 
instrument, orbit, and processing parameters which will be used in the following analyses.

\section{A. Coherence Estimation}

The key quantity in estimating the interferometric performance is the coherence [31], [32]

$$
\gamma_{\text {tot }}=\gamma_{\mathrm{SNR}} \cdot \gamma_{\mathrm{Quant}} \cdot \gamma_{\mathrm{Amb}} \cdot \gamma_{\mathrm{Rg}} \cdot \gamma_{\mathrm{Az}} \cdot \gamma_{\mathrm{Vol}} \cdot \gamma_{\mathrm{Temp}}
$$

where the right-hand side describes the different error contributions due to the limited SNR $\left(\gamma_{\mathrm{SNR}}\right)$, quantization $\left(\gamma_{\mathrm{Quant}}\right)$, ambiguities $\left(\gamma_{\mathrm{Amb}}\right)$, baseline decorrelation $\left(\gamma_{\mathrm{Rg}}\right)$, relative shift of the Doppler spectra $\left(\gamma_{\mathrm{Az}}\right)$, volume decorrelation $\left(\gamma_{\mathrm{Vol}}\right)$, and temporal decorrelation $\left(\gamma_{\text {Temp }}\right)$. In the following, each contribution will be discussed in more detail.

The finite sensitivity of each receiver causes a coherence loss [33]

$$
\gamma_{\mathrm{SNR}}=\frac{1}{\sqrt{\left(1+\mathrm{SNR}_{1}^{-1}\right) \cdot\left(1+\mathrm{SNR}_{2}^{-1}\right)}}
$$

where $\operatorname{SNR}_{\{1,2\}}$ is the SNR for each interferometric channel. Deviations between the two SNRs result from the relative shift of the antenna patterns (cf. Fig. 2), the performance degradation due to the delayed launch of the second satellite, and unequal noise figures resulting from different operating temperatures in the active $\mathrm{Tx} / \mathrm{Rx}$ and the passive $\mathrm{Rx}$-only receiver front ends. The actual SNR depends on the strength of the retuned radar signal and is derived from

$$
\operatorname{SNR}_{\{1,2\}}=\frac{\sigma_{0}\left(\theta_{\mathrm{i}}-\alpha\right)}{\operatorname{NESZ}_{\{1,2\}}\left(\theta_{\mathrm{i}}-\alpha\right)}
$$

where $\sigma_{0}$ is the normalized backscattering coefficient, and NESZ is the noise equivalent sigma zero level for each channel [34]

$$
\mathrm{NESZ}=\frac{4^{4} \pi^{3} r^{3} \nu \sin \left(\theta_{\mathrm{i}}-\alpha\right) k T B_{\mathrm{rg}} F L_{\mathrm{atm}} L_{\mathrm{sys}} L_{\mathrm{az}}}{P_{\mathrm{Tx}} G_{\mathrm{Tx}} G_{\mathrm{Rx}} \lambda^{3} c \tau_{\mathrm{p}} \mathrm{PRF}}
$$

where $r$ is the average transmit and receive range, $\nu$ is the satellite velocity, $\theta_{\mathrm{i}}$ is the incident angle, $\alpha$ is the local slope angle, $k$ is the Boltzmann constant, $T$ is the receiver temperature, $B_{\mathrm{rg}}$ is the bandwidth of the radar pulse, $F$ is the noise figure, $L_{\mathrm{sys}}$ denotes the system losses, $L_{\text {atm }}$ denotes the atmospheric losses, $P_{\mathrm{Tx}}$ is the transmit power, $G_{\mathrm{Tx}}$ and $G_{\mathrm{Rx}}$ are the gains of the transmit and receive antennas, respectively, $\lambda$ is the wavelength, $c$ is the velocity of light, $\tau_{\mathrm{p}}$ is the pulse duration, and PRF is the pulse repetition frequency. The azimuth losses $L_{\mathrm{az}}$ are derived from

$$
L_{\mathrm{az}}=\frac{\int\left|H_{\mathrm{proc}}(f)\right|^{2} \cdot d f}{\int\left|W\left(f ; \Delta f_{\mathrm{Dop}}\right)\right|^{2} \cdot\left|H_{\mathrm{proc}}(f)\right|^{2} \cdot d f}
$$

where $H_{\text {proc }}(f)$ is the transfer function of the azimuth processing filter, and $W\left(f ; \Delta f_{\text {Dop }}\right)$ is the weighting of the Doppler spectrum by the joint Tx-Rx azimuth antenna pattern. We assume, in the following, a rectangular shape for the envelope

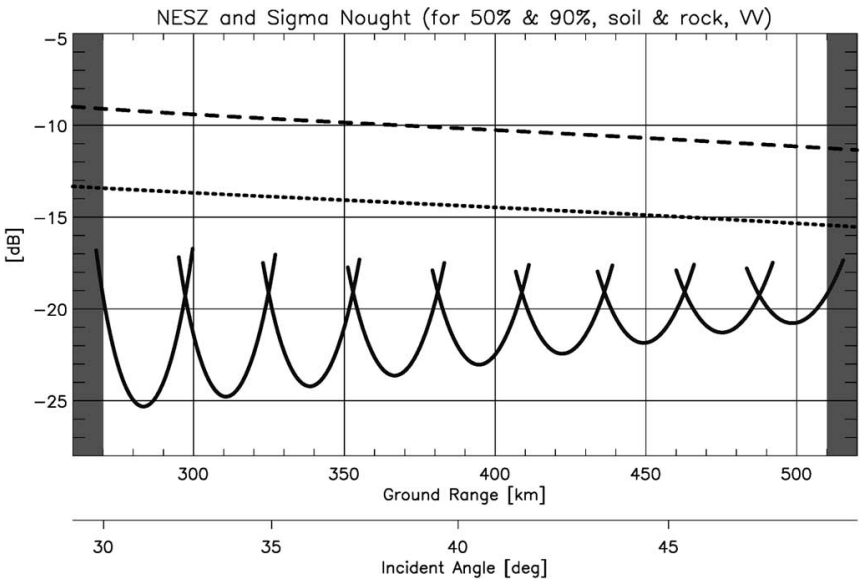

Fig. 8. NESZ for (solid) untapered stripmap mode and scattering coefficients for (dashed) $50 \%$ and (dotted) $90 \%$ occurrence levels.

of the azimuth processing filter $H_{\text {proc }}(f)$. The antenna patterns shown in Fig. 2 on the right then yield for the mono- and bistatic acquisition loss factors of $L_{\mathrm{az}}=1 \mathrm{~dB}$ and $L_{\mathrm{az}}=1.5 \mathrm{~dB}$, respectively. The bistatic loss factor rises to $L_{\mathrm{az}}=4.4 \mathrm{~dB}$ in case of an increase of the along-track displacement to $d_{\text {along }}=$ $2.5 \mathrm{~km}$, if the processed Doppler bandwidth remains unaltered. It is hence important to keep the along-track separation between the two satellites during the data acquisition as short as possible.

Fig. 8 shows the predicted NESZ of nine beams in the bistatic stripmap mode for an along-track displacement of $1 \mathrm{~km}$. The beams and their relative displacements have been obtained in an iterative optimization process such that they cover the required $240 \mathrm{~km}$ swath at the equator ${ }^{2}$ with a minimum incident angle of $\theta_{\mathrm{i}}=30^{\circ}$ and a constant height performance at the crossing points of adjacent subswaths (cf., Fig. 17). No amplitude tapering is used, and the chirp bandwidth is $B_{\mathrm{rg}}=$ $100 \mathrm{MHz}$ for all beams. For reference, Fig. 8 shows also the $\mathrm{X}$-band scattering coefficients for rock and soil surfaces at VV polarization and occurrence levels of (dashed) $50 \%$ and (dotted) $90 \%$, as provided in [30]. It can be seen that the SNR varies between $16 \mathrm{~dB}(11 \mathrm{~dB})$ and $8 \mathrm{~dB}(4 \mathrm{~dB})$ for an occurrence level of $50 \%(90 \%)$.

Another error source is the quantization of the recorded raw data signals [35]. The investigation of quantization errors is an important aspect, since the number of bits used for the digital representation of the recorded radar signals directly impacts the data rate to be transmitted to the ground. In a strict sense, the quantization errors have to be treated as a nonlinear and signal-dependent distortion, but for the current investigation, it is reasonable to approximate them as additive white Gaussian noise. This is justified by comparing the theoretical phase error predictions from the signal-to-quantization noise ratio (SQNR) to the interferometric phase errors obtained from a simulation using the complete TerraSAR-X block adaptive quantizer. Fig. 9 shows the relative increase of the

\footnotetext{
${ }^{2}$ The required swath width corresponds to the spatial separation of the TSX ground tracks and varies with latitude. TerraSAR-X has a sun-synchronous 11-day repeat cycle with 167 orbits, which yields a separation of $\sim 240 \mathrm{~km}$ between the adjacent ground tracks at the equator.
} 


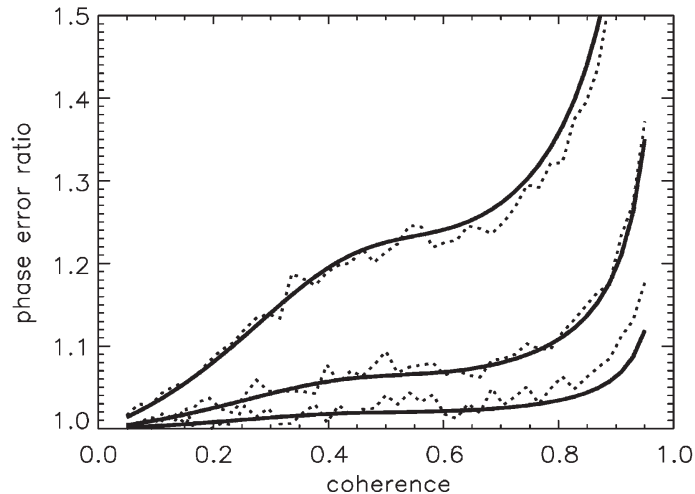

Fig. 9. Increase of the standard deviation of the interferometric phase errors due to quantization. Solid curves show from top to bottom the theoretic predictions of the relative phase error increase for an optimum uniform cartesian quantization [36] with two bit $\left(\mathrm{SQNR}=9.25 \mathrm{~dB} \leftrightarrow \gamma_{\text {Quant }}=0.894\right)$, three bit $\left(\mathrm{SQNR}=14.27 \mathrm{~dB} \leftrightarrow \gamma_{\text {Quant }}=0.964\right)$, and four bit $(\mathrm{SQNR}=$ $\left.19.38 \mathrm{~dB} \leftrightarrow \gamma_{\text {Quant }}=0.989\right)$. Dotted curves show the corresponding simulation results for the TSX BAQ.

interferometric phase errors for different quantization levels as a function of the coherence prior to quantization. The solid curves show the analytic predictions as derived from the SQNR for $n=15$ looks (cf., Section III-B), and the dotted lines show the corresponding simulation results. The analytic predictions from the coherence decrease match the simulation results quite well.

From this investigation, it becomes clear that the quantization errors could affect the interferometric performance in case of using a low bit rate. On the other hand, one has to take into account the limited downlink capacity of TanDEM-X, since the data from the two spacecrafts have to be transmitted to a ground station network which provides an average contact time of $\sim 10 \mathrm{~min} /$ orbit at a total net data rate $^{3}$ of $260 \mathrm{Mb} / \mathrm{s}$. For the selected sampling frequency ${ }^{4}$ of $110 \mathrm{MHz}$, this corresponds to average data acquisition times of $\sim 90 \mathrm{~s}$ per orbit in case of a quantization with $6 \mathrm{bits} / \mathrm{sample}$ and $180 \mathrm{~s}$ in case of a quantization with 3 bits/sample. For comparison, one global DEM acquisition with the beams defined in Fig. 8 will require, in total, somewhat less than $10^{6}$-s mapping time. For TerraSAR with its 5540 orbits per year, this corresponds then to an average acquisition time of $180 \mathrm{~s}$ per orbit if global coverage has to be achieved within one year. By taking into account the downlink bottleneck and the limited mission duration, one has to decide whether it is more appropriate to use a single acquisition with high data rate and low quantization errors or multiple acquisitions with lower data rate but higher quantization errors each. An answer to this question can be found by comparing the predicted phase errors from a single $n_{\text {bit }}$ data acquisition

$$
\varphi_{1}=\varphi\left[\gamma \cdot \gamma_{\mathrm{Quant}}\left(n_{\mathrm{bit}}\right), n_{\mathrm{look}}\right]
$$

\footnotetext{
${ }^{3}$ The close satellite formation excludes the simultaneous downlink, and the downlink time has to be shared among the spacecrafts.

${ }^{4}$ This value refers to complex I/Q sampling and is the lowest sampling frequency provided by TerraSAR-X [12].
}

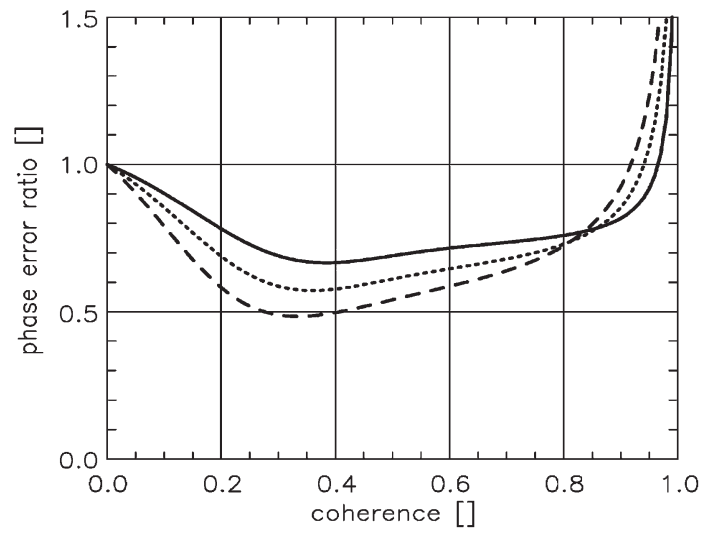

Fig. 10. Relative phase error standard deviations for different quantization strategies with a fixed total raw data volume. Shown are the phase error ratios $\varphi_{n} / \varphi_{1}$ for the following numbers of acquisitions: (solid) $n=2$, (dotted) $n=3$, and (dashed) $n=6$.

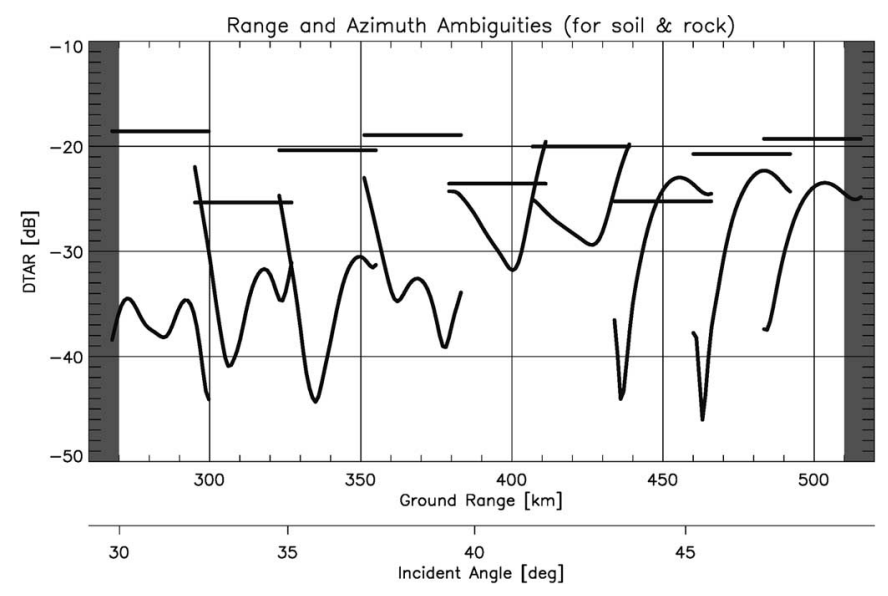

Fig. 11. Range and azimuth ambiguities in bistatic stripmap mode.

with the performance estimate for $n$ acquisitions with a quantization of $n_{\text {bit }} / n$ each $^{5}$

$$
\varphi_{n}=\varphi\left[\gamma \cdot \gamma_{\text {Quant }}\left(\frac{n_{\mathrm{bit}}}{n}\right), n \cdot n_{\mathrm{look}}\right]
$$

where $n_{\text {look }}$ is the number of independent looks, and $\gamma$ is the total coherence without quantization.

Fig. 10 shows the ratio $\varphi_{n} / \varphi_{1}$ for $n_{\text {bit }}=6, n_{\text {look }}=15$, and $n \in\{2,3,6\}$. By taking into account that the coherence for TanDEM-X ranges between 0.6 and 0.9 (cf., Fig. 13), it becomes clear that a significant reduction of the phase errors can be achieved by using two acquisitions with 3 bits/sample instead of a single acquisition with 6 bits/sample. A further reduction of the bit rate shows only a minor improvement (or even a degradation in case of high coherence). In the following, we will hence assume a block adaptive quantization with 3 bits/sample which allows for at least two global mappings within the nominal mission duration. This yields an SQNR of $\sim 14 \mathrm{~dB}$ and a coherence of $\gamma_{\text {Quant }} \approx 0.96$.

\footnotetext{
${ }^{5}$ This equation assumes a direct combination of the interferometric data from multiple acquisitions with equal baseline. A further improvement of the overall height accuracy can be obtained by selecting different baselines for the different acquisitions. This will be analyzed in Section III-C.
} 
The third component in (19) represents the error contributions from distributed range and azimuth ambiguities. The corresponding coherence loss can be approximated by

$$
\gamma_{\mathrm{Amb}}=\frac{1}{1+\mathrm{RASR}} \cdot \frac{1}{1+\mathrm{AASR}}
$$

where RASR and AASR are the range and azimuth ambiguityto-signal ratios, respectively. Fig. 11 shows the predicted range and azimuth ambiguities for the individual swaths in the bistatic stripmap mode. The corresponding coherence loss will hence vary among the swaths with $\gamma_{\mathrm{Amb}}>0.97$.

The fourth component in (19) represents coregistration errors in range as well as baseline decorrelation due to the imaging of a flat surface from slightly different incident angles [37]. As already mentioned in Section II-A, baseline decorrelation can be avoided by filtering the two object spectra to a common frequency band [18]. This reduces the range resolution and, thereby, the number of independent looks for a given postspacing, which will be taken into account in the estimation of the interferometric phase errors (cf., Section III-B). By assuming an unweighted processing of the rectangular range spectrum, we then obtain $\gamma_{\operatorname{Rg}}=\operatorname{sinc}(\pi \cdot \partial r)$, where $\partial r$ is the relative range shift between the two interferometric SAR images in fractions of a resolution cell [31]. A misregistration of $10 \%$ will hence cause a coherence loss of $\gamma_{\mathrm{Rg}}=0.984$.

The fifth component in (19) accounts for the coherence loss from a misregistration in azimuth as well as a Doppler shift between the two interferometric channels [33]. Different Doppler spectra may, for example, originate from a nonvanishing along-track displacement if the two azimuth antenna patterns are steered to the same joint antenna footprint, as shown in the left-hand side of Fig. 2. The coherence loss is then derived as

$$
\gamma_{\mathrm{Az}}=\frac{\int H_{1}(f) \cdot H_{2}^{*}(f) \cdot \exp \left(-j \cdot 2 \pi \cdot \delta \tau_{\mathrm{az}} \cdot f\right) \cdot d f}{\sqrt{\int\left|H_{1}(f)\right|^{2} \cdot d f} \cdot \sqrt{\int\left|H_{2}(f)\right|^{2} \cdot d f}}
$$

where $\delta \tau_{\mathrm{az}}=\delta \mathrm{az} / \nu_{\mathrm{grd}}$ is the coregistration error in azimuth, and the azimuth weighting functions $H_{1}(f)$ and $H_{2}(f)$ are given by the product of the joint antenna patterns with the spectral transfer function of the azimuth processing filter $H_{\text {proc }}(f)$

$$
\begin{aligned}
& H_{1}(f)=A_{\mathrm{Tx}}(f) \cdot A_{\mathrm{Rx}, 1}(f) \cdot H_{\mathrm{proc}}(f) \\
& H_{2}(f)=A_{\mathrm{Tx}}(f) \cdot A_{\mathrm{Rx}, 2}(f) \cdot H_{\mathrm{proc}}(f) .
\end{aligned}
$$

The effect of Doppler decorrelation can be minimized by confining the support region of the azimuth processing filter $H_{\text {proc }}(f)$ to those frequencies where the two azimuth spectra have similar magnitude. This strategy was also applied for the definition of the common Doppler spectrum on the left-hand side of Fig. 2, which yields $\gamma_{\mathrm{Az}}=0.976$ for $\delta \mathrm{az}=0.3 \mathrm{~m}$. This value drops to $\gamma_{\mathrm{Az}}=0.913$ for an along-track displacement of $2.5 \mathrm{~km}$ if we keep the processed Doppler bandwidth constant. For comparison, the independent zero Doppler steering on the right-hand side of Fig. 2 yields an azimuth decorrelation of

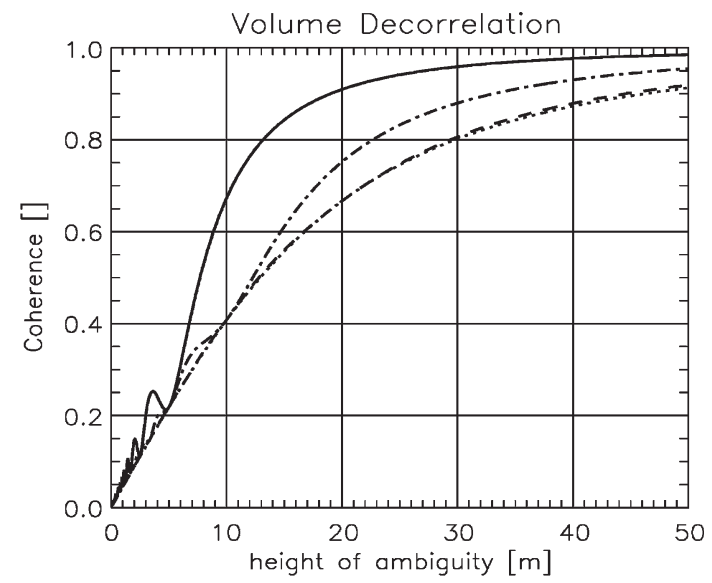

Fig. 12. Coherence loss from volume decorrelation for an extinction of $1.0 \mathrm{~dB} / \mathrm{m}$. The volume heights are (solid) 5, (dashed-dotted) 10, (dashed) 20, and (dotted) $40 \mathrm{~m}$.

$\gamma_{\mathrm{Az}}=0.989$ which is (almost) independent of the along-track separation.

The last but one term on the right-hand side of (19) describes the coherence loss due to volume scattering in vegetated areas [15]. Similar to the classical baseline decorrelation, the effect of volume scattering can be understood as an increase of the interferometric phase uncertainty resulting from the existence of multiple scatterers within a single resolution cell. Each of these scatterers has a different height $z$ and will hence contribute with a different interferometric phase $\varphi=2 \pi z / h_{\mathrm{amb}}$. The coherence is then derived from the ensemble average over all scatterer distributions

$\gamma_{\mathrm{Vol}}=\int_{0}^{h_{\nu}} \sigma^{0}(z) \cdot \exp \left(j 2 \pi \frac{z}{h_{\mathrm{amb}}}\right) \cdot d z / \int_{0}^{h_{\nu}} \sigma^{0}(z) \cdot d z$

where $\sigma^{0}(z)$ represents the vertical scatterer profile. To model $\sigma^{0}(z)$, we note first that, for the vegetated areas, the effective scattering cross section will be a function of the penetration of the electromagnetic wave into the vegetation layer. By taking into account the extinction in a homogeneous medium, we may assume a vertical scattering profile

$$
\sigma^{0}(z)=\exp \left[-2 \cdot \beta \cdot \frac{h_{\nu}-z}{\cos \left(\theta_{\mathrm{i}}\right)}\right], \quad 0 \leq z<h_{\nu}
$$

where $h_{\nu}$ is the vegetation height, and $\beta$ is the one-way amplitude extinction coefficient in Nepers per meter. Fig. 12 shows the coherence loss for volume heights of 5, 10, 20, and $40 \mathrm{~m}$ and an extinction of $1.0 \mathrm{~dB} / \mathrm{m}$ (corresponding to $\beta=0.115 \mathrm{~Np} / \mathrm{m}$ ) as a function of the height of ambiguity for an incident angle of $35^{\circ}$. It becomes clear that volume scattering can have a significant impact on the interferometric coherence in areas with tall vegetation. The increased "phase noise" in case of a low height of ambiguity may furthermore cause problems with phase unwrapping. In order to avoid such errors, we will try to keep the height of ambiguity in the HELIX formation as high as possible while still meeting the HRTI-3 requirements. The potential impact of volume 
Total Coherence (soil \& rock, W

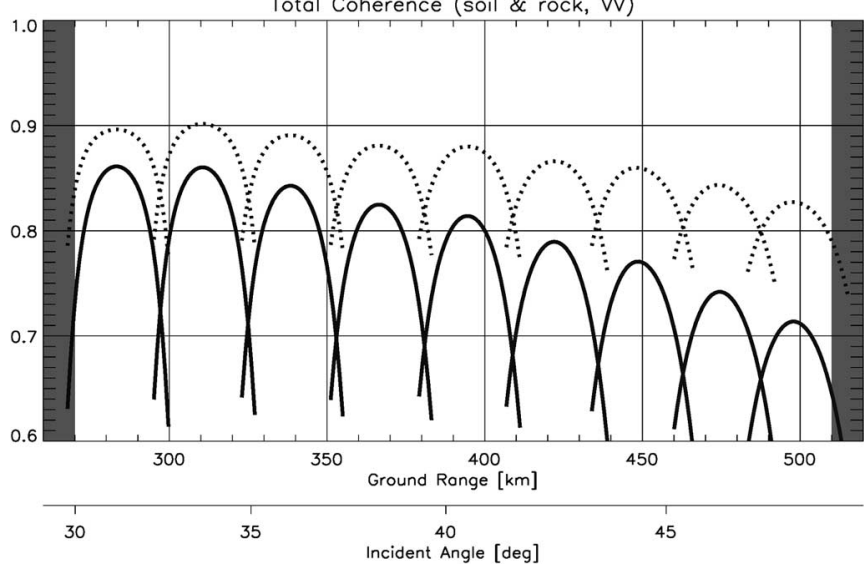

Fig. 13. Total coherence predicted for bistatic stripmap mode. The individual parabola-like curves show the variation of the coherence within each stripmap swath. The dotted and solid lines are for the $50 \%$ and $90 \%$ occurrence levels of the backscatter coefficients.

decorrelation on the TanDEM-X performance can be mitigated by increasing the independent postspacing in the vegetated areas. Such areas are systematically identified from the recorded data by evaluating the changes of the local coherence. For this, the residual highly predictable decorrelation sources in (19) will be estimated by taking into account the amplitude of the focused SAR signal. A comparison with the coherence magnitude of the recorded data will then allow a direct measurement of volume decorrelation in case of short along-track baselines. This technique is hence complementary to the systematic estimation of temporal decorrelation, as suggested in [32]. The comparison of repeat- and single-pass interferograms will furthermore allow for a clear separation of the otherwise intermixed factors from volume and temporal decorrelation, which allows for an improved characterization of the 3-D scattering process. The measured coherence loss from volume (and temporal) decorrelation can moreover be used to reduce the height bias in the vegetated areas.

The last term in (19) describes the errors from a change of the scatterer structure between the acquisition of the two interferometric channels with equal Doppler frequencies. We will, in the following, neglect any coherence loss due to temporal decorrelation. While such an assumption seems to be justified for most types of land cover, it might cause severe problems in dynamic oceanographic mapping if the along-track distance between the receiver satellites exceeds hundred meters (cf., Section V-A). Temporal decorrelation and systematic phase shifts from ocean surface currents may also limit the usability of ocean data takes for height calibration. Rather short decorrelation times on the order of $50 \mathrm{~ms}$ and less, which are corresponding to an along-track separation below $750 \mathrm{~m}$, have furthermore been observed for some types of vegetation imaged in the X-band at moderate to high wind speeds [38]. It is hence of great importance to keep the along-track separation between the two satellites in such areas as short as possible. The along-track displacements on the order of $100 \mathrm{~m}$ and less (corresponding to a time delay of $\tau_{\mathrm{d}}<7 \mathrm{~ms}$ ) can be achieved without any collision risk between the two satellites owing to the HELIX formation, which enables a systematic adjustment of the optimum along-track baseline for a specified latitude range.

Fig. 13 shows the total coherence $\gamma_{\text {tot }}$ for TanDEM-X operating in standard stripmap mode in case of surface scattering. The dotted curves show the estimated coherence for the scattering coefficients with an occurrence level of $50 \%$. The coherence is on the order of 0.8 to 0.9 . The solid lines are for scattering coefficients corresponding to an occurrence level of $90 \%$. The lower scattering reduces the coherence particularly at higher incident angles, where the limited SNR becomes the dominant error source.

\section{B. Interferometric Phase Errors}

The knowledge of the total coherence $\gamma_{\text {tot }}$ allows now for the derivation of the interferometric phase errors. The probability density function (pdf) of the phase difference $p_{\varphi}(\varphi)$ between the two interferometric SAR channels is given by [39]

$$
\begin{aligned}
& p_{\varphi}(\varphi)=\frac{\Gamma\left(n+\frac{1}{2}\right)\left(1-\gamma_{\text {tot }}^{2}\right)^{n} \gamma_{\text {tot }} \cos \varphi}{2 \sqrt{\pi} \Gamma(n)\left(1-\gamma_{\text {tot }}^{2} \cos ^{2} \varphi\right)^{n+\frac{1}{2}}} \\
& +\frac{\left(1-\gamma_{\text {tot }}^{2}\right)^{n}}{2 \pi} F\left(n, 1 ; \frac{1}{2} ; \gamma_{\text {tot }}^{2} \cos ^{2} \varphi\right)
\end{aligned}
$$

where $n$ is the number of independent looks, $\Gamma$ is the gamma function, and $F$ is the Gauss hypergeometric function [40]. The standard deviation of the interferometric phase errors is then given by

$$
\sigma_{\varphi}=\sqrt{\int_{-\pi}^{\pi} \varphi^{2} p_{\varphi}(\varphi) \cdot d \varphi}
$$

The left-hand side of Fig. 14 shows the well-known dependence of $\sigma_{\varphi}$ on the coherence $\gamma_{\text {tot }}$ for different look numbers $n$ [32].

An estimation of the $90 \%$ point-to-point height errors, as required by HRTI-3, requires the computation of the difference between two random variables where each describes the fluctuation of the interferometric phase at one location. The pdf of this difference corresponds to a convolution between the two pdfs $p_{\varphi}(\varphi)$ [41]. The $90 \%$ point-to-point phase error $\varphi_{90 \%}$ is then obtained from

$$
\int_{-\varphi_{90 \%}}^{\varphi_{90 \%}}\left[p_{\varphi}(\varphi) \otimes p_{\varphi}(\varphi)\right] \cdot d \varphi=0.9
$$

where $\otimes$ denotes convolution, and $p_{\varphi}(\varphi)$ is the pdf given in (31). The right-hand side of Fig. 14 shows the unwrapped multilook phase error $\varphi_{90 \%}$ as a function of the coherence for different look numbers. Note the different shapes for the standard deviation and the $90 \%$ point-to-point error curves as a result of the varying shape of the non-Gaussian phase error pdfs. 

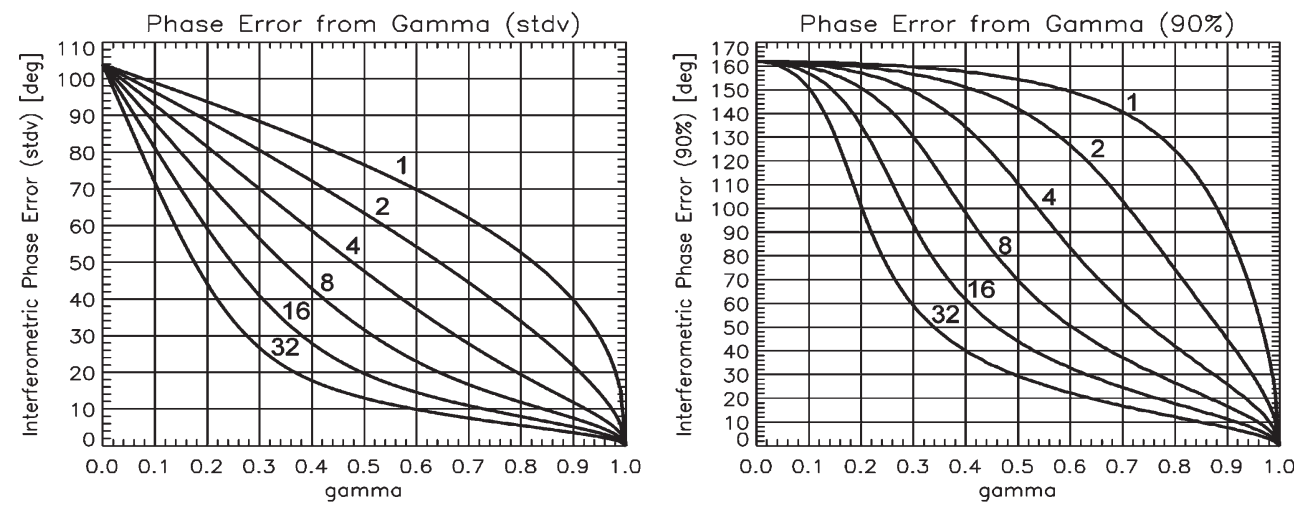

Fig. 14. (Left) Standard deviation and (right) $90 \%$ point-to-point errors of the interferometric phase. The look numbers are indicated above each curve.

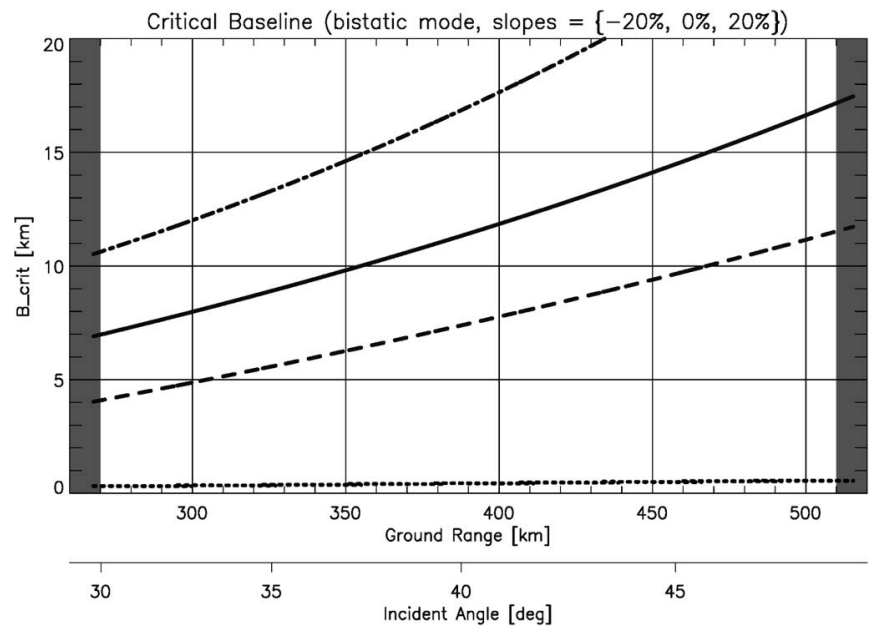

Fig. 15. Critical baseline for the TanDEM-X operating with a chirp bandwidth of $100 \mathrm{MHz}$ for slopes of (solid) $0 \%$, (dashed) $-20 \%$, and (dashed-dotted) $+20 \%$. The dotted line on the bottom shows the perpendicular baseline corresponding to a height of ambiguity of $30 \mathrm{~m}$.

To compute the number of independent looks $n$, we recall first that range filtering has been assumed to optimize the interferometric coherence. As a result, the common bandwidth may become lower than that of each individual channel, thereby reducing the number of independent looks for a fixed independent postspacing. The number of looks in range can be approximated by ${ }^{6} n_{\mathrm{rg}}=\Delta \mathrm{rg} / \delta \mathrm{rg}$, where $\Delta \mathrm{rg}$ is the postspacing of the final product in range, and $\delta \mathrm{rg}$ is the ground range resolution within each channel after spectral filtering. By assuming an unweighted range focusing, the latter can be computed from [20]

$$
\delta \mathrm{rg}=\frac{c \cdot \cos (\alpha)}{2 \cdot B_{\mathrm{rg}} \cdot \sin \left(\theta_{\mathrm{i}}-\alpha\right)} \cdot \frac{B_{\perp, \mathrm{crit}}}{B_{\perp, \mathrm{crit}}-B_{\perp}}
$$

where $B_{\perp}$ is the interferometric baseline perpendicular to the line of sight, and $B_{\perp, \text { crit }}$ is the critical baseline which is given by [32]

$$
B_{\perp, \mathrm{crit}}=\frac{2 \cdot B_{\mathrm{rg}} \cdot \lambda \cdot r \cdot \tan \left(\theta_{\mathrm{i}}-\alpha\right)}{c} .
$$

\footnotetext{
${ }^{6}$ This formula underestimates the number of effective looks if we assume spatial smoothing of the interferograms with a rectangular convolution kernel of extension $\Delta \mathrm{rg}$. A more rigorous formula can be obtained in analogy to (36).
}

Fig. 15 shows the critical baseline of TanDEM-X for different local slopes of the imaged scene. The chirp bandwidth in this example is $100 \mathrm{MHz}$. The dotted line in Fig. 15 shows, for comparison, the typical baseline lengths which will be used for the operational DEM generation with TanDEM-X (cf., Section IV). It becomes clear that TanDEM-X will only use a small fraction of the critical baseline, and the loss in range resolution from spectral filtering will be less than $10 \%$.

The computation of the effective number of azimuth looks is more involved, since we have to take into account the nonrectangular and, in general, the different joint azimuth antenna patterns which will introduce some correlation in the interferometric data samples. This will then reduce the effective number of looks if compared to an ideal rectangular transfer function [42]. The effective number of azimuth looks for interferometric processing can be approximated by (36), shown at the bottom of the next page, where $H_{\text {ant }}(f), H_{\text {proc }}(f)$, and $H_{\text {smooth }}(f)$ are the spectral transfer functions of the joint antenna patterns, the processing filters, and the smoothing filter, respectively. In the following, we assume spatial averaging of the interferogram with a rectangular convolution kernel of azimuth extension $\Delta \mathrm{az}=12 \mathrm{~m}$, which has the transfer function $H_{\text {smooth }}=\operatorname{sinc}\left(f \cdot \Delta \mathrm{az} / \nu_{\text {grd }}\right)$, where $\nu_{\text {grd }}=7.1 \mathrm{~km} / \mathrm{s}$ is the beam velocity on the ground. In case of an unweighted azimuth processing with the bandwidths and antenna patterns indicated on the left- and right-hand sides of Fig. 2, we obtain for the effective number of azimuth looks values of $n_{\mathrm{az}}=3.8$ and $n_{\mathrm{az}}=$ 4.1 , respectively. These values improve to $n_{\mathrm{az}}=4.4$ for an along-track displacement of $0 \mathrm{~m}$, while an increase of the along-track displacement to $2.5 \mathrm{~km}$ reduces them to $n_{\mathrm{az}}=2.2$ in case of individual zero Doppler steering. It is hence clear that the along-track displacement between the two satellites should be kept as small as possible (cf. the Appendix).

By assuming that the maximum along-track displacement in the HELIX formation stays always below $1 \mathrm{~km}$, the total number of independent looks varies between approximately $n=15$ for an incident angle of $30^{\circ}$ and $n=23$ for an incident angle of $48^{\circ}$ in case of flat terrain and the HRTI- 3 postspacing of $12 \mathrm{~m} \times 12 \mathrm{~m}$. A reduction of the postspacing to $6 \mathrm{~m} \times$ $6 \mathrm{~m}$, as, for example, required by the HRTI- 4 standard, may reduce the number of looks to values below four which leads to an unfavorable coherence to phase scaling, as shown in the right-hand side of Fig. 14. For the generation of customized 


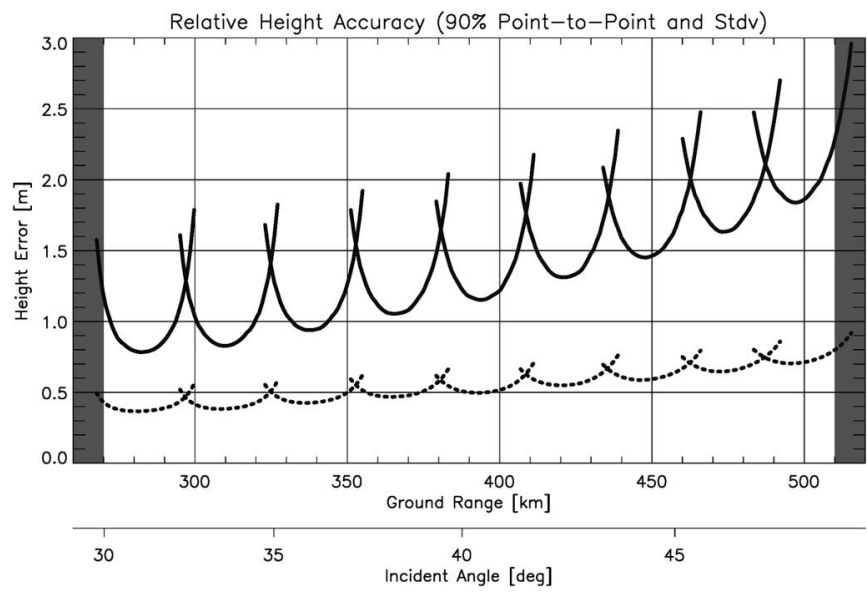

Fig. 16. Relative height accuracy for an effective baseline of $500 \mathrm{~m}$ in the stripmap mode. (Solid) 90\% point-to-point height errors. (Dotted) Standard deviation.

DEMs with improved spatial resolution on a local scale, which represents a secondary mission goal of TanDEM-X, it is hence advisable to increase the chirp bandwidth from 100 to $150 \mathrm{MHz}$ or even $300 \mathrm{MHz}$, where the latter is provided by TerraSAR-X on an experimental basis [12].

\section{Relative Height Accuracy}

The relative height errors may now be derived from

$$
\Delta h=h_{\mathrm{amb}} \cdot(\Delta \varphi / 2 \pi)
$$

where $\Delta \varphi$ is given by either $\sigma_{\varphi}$ or $\varphi_{90 \%}$. Fig. 16 shows the predicted height accuracy for a fixed effective baseline of $500 \mathrm{~m}$. The solid lines indicate the $90 \%$ point-to-point height errors, and the dotted lines indicate the corresponding standard deviation. This and the following performance examples include an additional phase error of $5^{\circ}$ to account for residual synchronization and instrument phase errors which may cause a low-frequency height modulation of the resulting DEM.

The height errors in Fig. 16 show a significant increase from near to far ranges. One reason for this increase is the systematic decrease of the phase-to-height scaling which is corresponding to a systematic increase of the height of ambiguity with increasing incident angles. As TanDEM-X enables a flexible selection of the interferometric baseline, it is hence advisable to adapt the length of the baselines to a fixed height of ambiguity. The derivation of suitable HELIX formations with an almost constant height of ambiguity will be explained in Section IV.

A further improvement of the DEM accuracy can be achieved by combining the overlapping data segments from successive TanDEM-X satellite passes. The redundant interferometric signals from overlapping beams can then be used to partially

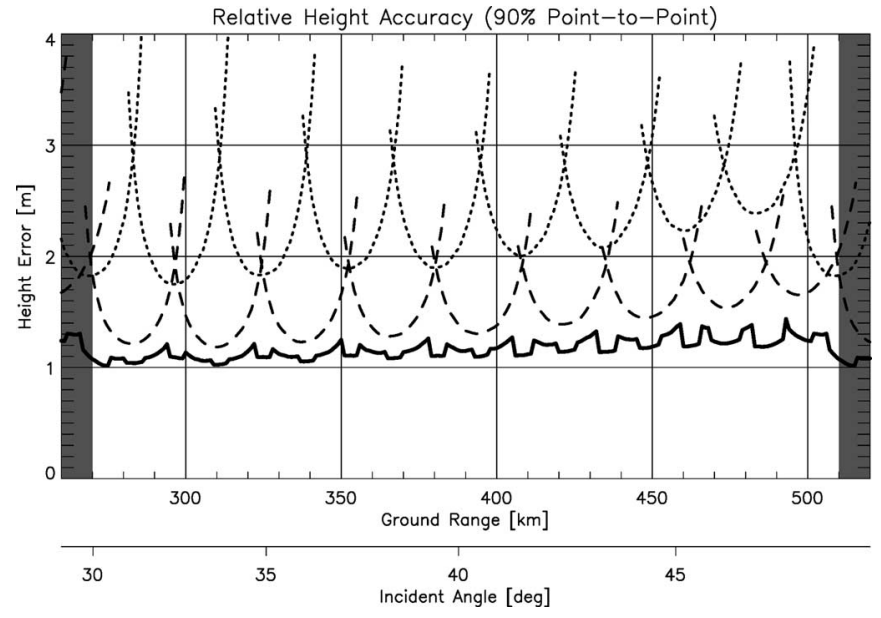

Fig. 17. Height accuracy for a height of ambiguity of (dotted) 45 and (dashed) $30 \mathrm{~m}$. The solid curve shows the error resulting from the combination of multiple swaths. All errors are point-to-point height errors for a $90 \%$ confidence interval.

compensate the performance decay at each swath border and to improve, thereby, the overall interferometric height accuracy. By assuming a linear combination of $N$ height estimates

$$
\tilde{h}_{\mathrm{comb}}=\sum_{i=1}^{N} \alpha_{i} \cdot \tilde{h}_{i}
$$

the weights $\alpha_{i}$ can be obtained by minimizing the overall height error under the assumption of additive and mutually uncorrelated height errors with known variances $\Delta h_{i}^{2}$ which yields

$$
\alpha_{i}=\Delta h_{i}^{-2} \cdot\left(\sum_{k=1}^{N} \Delta h_{k}^{-2}\right)^{-1} .
$$

The overall height error is finally derived by inserting (39) into (38) as

$$
\Delta h_{\mathrm{comb}}=\sqrt{\prod_{i=1}^{N} \Delta h_{i}^{2} / \sum_{i=1}^{N}\left(\frac{1}{\Delta h_{i}^{2}} \cdot \prod_{k}^{N} \Delta h_{k}^{2}\right)} .
$$

Fig. 17 shows the predicted point-to-point height errors for the $90 \%$ confidence interval, assuming two sets of DEM data acquisitions with two fixed heights of ambiguity of (dashed) $30 \mathrm{~m}$ and (dotted) $45 \mathrm{~m}$. Note that the two acquisition sets use mutually displaced beams to further improve the performance. The height error from the combination of all acquisitions is shown in solid style, yielding an almost constant performance with an accuracy which is well below the 2-m requirement.

The impacts of slopes and volume decorrelation on the achievable performance are shown in Fig. 18. The figure on the

$$
n_{\mathrm{az}}=\frac{\iint H_{\mathrm{proc}}^{2}\left(f_{1}\right) \cdot H_{\mathrm{proc}}^{2}\left(f_{2}\right) \cdot\left[H_{\mathrm{ant}}^{2}\left(f_{1}\right)+H_{\mathrm{ant}}^{2}\left(f_{2}\right)\right] \cdot d f_{1} \cdot d f_{2}}{\iint H_{\mathrm{proc}}^{2}\left(f_{1}\right) \cdot H_{\mathrm{proc}}^{2}\left(f_{2}\right) \cdot\left[H_{\mathrm{ant}}^{2}\left(f_{1}\right)+H_{\mathrm{ant}}^{2}\left(f_{2}\right)\right] \cdot H_{\mathrm{smooth}}^{2}\left(f_{2}-f_{1}\right) \cdot d f_{1} \cdot d f_{2}}
$$



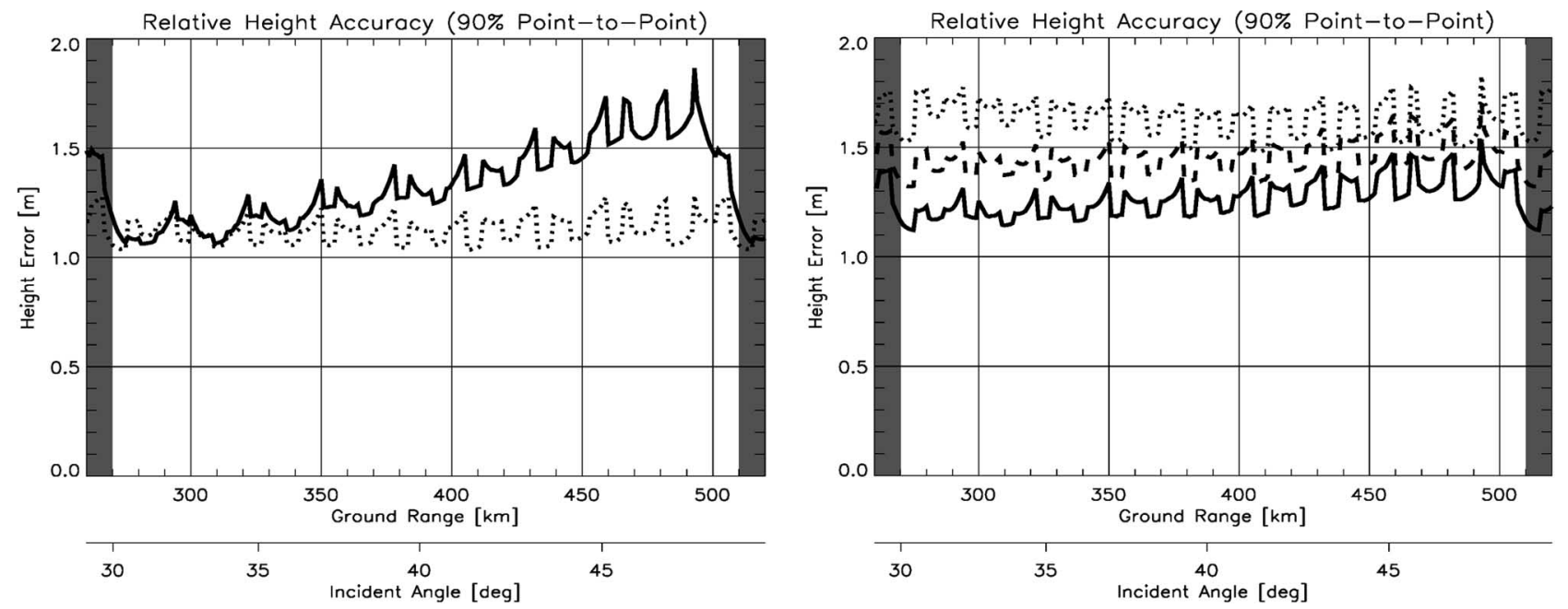

Fig. 18. Impact of slopes and volume scattering on the relative height errors. (Left) Height accuracy for a local slope of 20\% (dotted) facing toward and (solid) away from the radar. (Right) Height accuracy for volume heights of (solid) 5, (dashed) 10, and (dotted) $20 \mathrm{~m}$.

left shows that a slope of $20 \%$ facing away from the radar may increase the height errors particularly at high incident angles. This is mainly due to the lower scattering for shallow incident angles. The reduced spectral overlap for slopes facing toward the radar has only a minor impact and is partially compensated by the increased scattering. The figure on the right-hand side shows the impact of volume decorrelation for vegetation heights of 5,10 , and $20 \mathrm{~m}$ for an extinction of $1 \mathrm{~dB} / \mathrm{m}$. It is clear that volume scattering will impact the performance for all incident angles, but this effect is mitigated by the availability of two interferometric baselines.

\section{Baseline Estimation Errors}

In the previous derivations, we have neglected the errors due to the finite accuracy of relative baseline estimation. Such errors will mainly cause a low-frequency modulation of the DEM, thereby simultaneously contributing to the relative and absolute height errors. For the latter, the HRTI-3 standard is much less stringent and requires an accuracy of $10 \mathrm{~m}$ at a $90 \%$ confidence level.

Baseline estimation errors can be divided into along-track, cross-track, and radial errors. The along-track errors will be sufficiently resolved during the coregistration and are hence regarded as uncritical. The cross-track and radial errors may cause errors in both the line of sight $\left(\Delta B_{\|}\right)$and perpendicular $\left(\Delta B_{\perp}\right)$ to the line of sight. The baseline errors perpendicular to the line of sight will cause a bias in the phase-to-height scaling. The resulting height error is given by

$$
\Delta h=h \cdot \frac{\Delta B_{\perp}}{B_{\perp}}
$$

where $h$ is the topographic height, $\Delta B_{\perp}$ is the error of the baseline estimate perpendicular to the line of sight, and $B_{\perp}$ is the length of the perpendicular baseline. By assuming a maximum topographic height of $h=9000 \mathrm{~m}$ and baselines corresponding to a height of ambiguity of $h_{\mathrm{amb}}=35 \mathrm{~m}$ (i.e., $B_{\perp}=260 \mathrm{~m}$ for $\theta_{\mathrm{i}}=30^{\circ}$ and $B_{\perp}=439 \mathrm{~m}$ for $\theta_{\mathrm{i}}=45^{\circ}$ ),
TABLE III

HEIGHT ERRORS FOR 1-mm BASELINE ESTIMATION UNCERTAINTY

\begin{tabular}{|c|c|c|c|c|}
\hline \multirow{3}{*}{ Incident Angle } & \multirow{3}{*}{$\begin{array}{c}\text { Normal } \\
\text { Baseline } \\
\left(h_{a m b}=35 \mathrm{~m}\right)\end{array}$} & \multicolumn{3}{|c|}{ Height Errors (for $h_{a m b}=35 \mathrm{~m}$ ) } \\
\hline & & \multicolumn{2}{|c|}{$\Delta B_{\| /}=1 \mathrm{~mm}$} & $\Delta B_{\perp}=1 \mathrm{~mm}$ \\
\hline & & $\Delta t$ & $\Delta h / \Delta s$ (tilt) & $\Delta /(\mathrm{h}=9 \mathrm{~km})$ \\
\hline $30^{\circ}$ & $260 \mathrm{~m}$ & \multirow{2}{*}{$1.1 \mathrm{~m}$} & $3.8 \mathrm{~mm} / \mathrm{km}$ & $3.5 \mathrm{~cm}$ \\
\hline $45^{\circ}$ & $439 \mathrm{~m}$ & & $2.3 \mathrm{~mm} / \mathrm{km}$ & $2.1 \mathrm{~cm}$ \\
\hline
\end{tabular}

a baseline estimation error of $\Delta B_{\perp}= \pm 1 \mathrm{~mm}$ will result in height errors of $\pm 3.5 \mathrm{~cm}$ and $\pm 2.1 \mathrm{~cm}$ for incident angles of $\theta_{\mathrm{i}}=30^{\circ}$ and $\theta_{\mathrm{i}}=45^{\circ}$, respectively.

Errors in the relative position estimates of the antenna phase centers parallel to the line of sight $\left(\Delta B_{/ /}\right)$will primarily cause a rotation of the reconstructed DEM about the (master) satellite position. As a result, the DEM will be vertically displaced by

$$
\Delta h=r \cdot \sin \left(\theta_{\mathrm{i}}\right) \cdot \frac{\Delta B_{\|}}{B_{\perp}}=\frac{h_{\mathrm{amb}}}{\lambda} \cdot \Delta B_{\|}
$$

where $r$ and $\theta_{\mathrm{i}}$ are the slant range distance and the incident angle of an appropriately selected reference point (e.g., at midswath), respectively. This vertical displacement will be $\Delta h= \pm 1.1 \mathrm{~m}$ for $\Delta B_{/ /}= \pm 1 \mathrm{~mm}$ and $h_{\mathrm{amb}}=35 \mathrm{~m}$. A parallel baseline error will furthermore cause a tilt of the DEM which is given by

$$
\varphi_{\mathrm{tilt}}=\frac{\Delta h}{\Delta s}=\frac{\Delta B_{\|}}{B_{\perp}}
$$

where $\Delta s$ is the ground range distance from the selected reference point. The resulting tilt will be 3.8 and $2.3 \mathrm{~mm} / \mathrm{km}$ for incident angles of $\theta_{\mathrm{i}}=30^{\circ}$ and $\theta_{\mathrm{i}}=45^{\circ}$, respectively $\left(\Delta B_{/ /}=1 \mathrm{~mm}\right.$ and $\left.h_{\mathrm{amb}}=35 \mathrm{~m}\right)$. Table III summarizes the predicted height errors resulting from $\Delta B_{/ /}=1 \mathrm{~mm}$ and $\Delta B_{\perp}=1 \mathrm{~mm}$.

Precise baseline determination will be performed by a double differential evaluation of the GPS carrier phase measurements. Analyses based on the experience gained from the GRACE mission indicate an achievable accuracy for the estimation of 


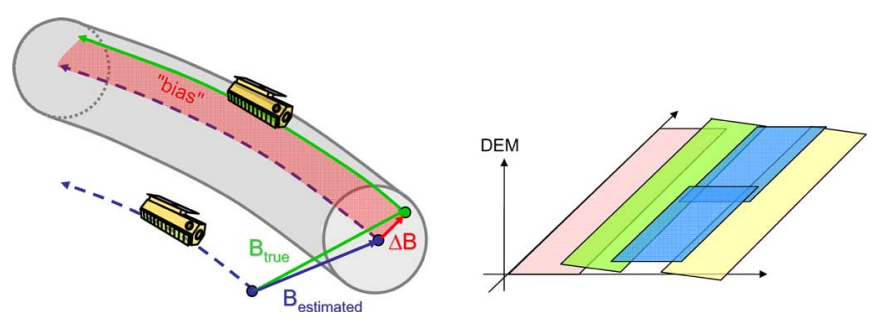

Fig. 19. Illustration of the major impact of baseline estimation errors. (Left) Unknown baseline offset during each data take. The baseline measurement accuracy of 1-2 mm is indicated by the gray tube. (Right) Vertical displacement and tilt of adjacent swaths as a result of different baseline offsets during the data takes.

relative satellite positions on the order of $1-2 \mathrm{~mm}$ [44]. In comparison, variations of both the GPS and the SAR antenna phase centers can be neglected since they affect both satellites in almost the same manner. An uncertainty in the attitude of the two satellites will cause additional baseline errors that stay well below $1 \mathrm{~mm}$. Furthermore, note in this context that both satellites experience almost the same gravity field and are exposed to highly correlated orbital perturbations. Residual (i.e., unmodeled) variations of the overall baseline vector will hence show a high degree of temporal correlation. Even in the case of a large differential acceleration of $\Delta a=100 \cdot 10^{-9} \mathrm{~m} / \mathrm{s}^{2}$ (e.g., due to unmodeled differential drag between the two satellites, etc.), the resulting differential orbit error after a $100-\mathrm{km}$ data take will be on the order of only $10 \mu \mathrm{m}$. By noting furthermore that such an acceleration will mainly affect the estimates of the along-track baseline (which are uncritical for the crosstrack interferometry), we may conclude that residual orbit fluctuations can be neglected in the computation of relative height errors (the area for relative point-to-point height errors in HRTI-3 is approximately $100 \mathrm{~km} \times 100 \mathrm{~km}$ ). This shows the great advantage of using an unperturbed free-flying satellite formation in contrast to a single-pass SAR interferometer employing a mechanical connection between the two SAR antennas by either a long boom [7] or a tether [45]. Both solutions imply additional mechanical forces within the differential gravity field and lead to annoying baseline oscillations that are difficult to correct for by the final calibration.

A factor not to be ignored is the uncertainty in the relative RF phase centre positions at the beginning of the data take which may result in a tilt of the acquired DEM swaths as illustrated in Fig. 19. For example, an initial error in the estimate of the SAR relative phase center position of $\Delta B_{/ /}= \pm 1 \mathrm{~cm}$ could, in the worst case (assuming the unlikely case of four equally tilted swaths), result in a relative height error of $\pm 3.8 \mathrm{~m}$ for $\Delta s=$ $100 \mathrm{~km}$. Such a tilt can be reduced by additional calibration data takes from crossing orbits via a bundle block adjustment in either radar or DEM geometry [46]. Appropriate data takes will be acquired during the third mission year after a $180^{\circ}$ rotation of the eccentricity vector in the HELIX formation, thereby taking advantage of an increased baseline length in combination with the advanced interferometric modes (alternating bistatic and simultaneous transmit). A promising supplement-or even a complete alternative-is the use of ICESat data which provide a dense grid of global elevation measurements with submeter accuracy [47]. If necessary, one could in addition employ the following: 1) a sparse set of calibration targets and reference points; 2) dynamic GPS tracks on the ground; 3) laser and radar altimeter data from other spaceborne missions; and 4) ocean data takes acquired with TanDEM- $X$ using short along-track baselines. By taking into account that the formation flight with TanDEM-X allows for the acquisition of long data takes with high phase and baseline stability, ${ }^{7}$ it becomes clear that the absolute DEM accuracy is mainly limited by the accuracy of the calibration sources. Therefore, the 10-m absolute height accuracy, as required by HRTI-3, may well be achieved.

\section{Optimization of the Data AcQuisition Plan}

The performance analyses in Section III-C revealed that the derivation of a global DEM according to the HRTI-3 standard requires data acquisitions with a height of ambiguity on the order of 30 to $40 \mathrm{~m}$. As the height of ambiguity depends not only on the length of the cross-track baseline and its orientation in space but also on the actual slant range and incident angle within the swath to be imaged, a single HELIX formation will not be sufficient to monitor a wide coverage range. Therefore, a small set of HELIX formations will be employed for global DEM data acquisition. Each individual HELIX is then used to map a subset of the required swaths which depends, in turn, on the geographic latitude $\phi$. An optimized set of HELIX formations with its associated swath assignment can be derived by minimizing the overall variation of the height of ambiguity within a predefined latitude range. This minimization is achieved in a two-step procedure where we first derive the optimum swath assignment for a given set of HELIX formations $\left\{H_{1}, \ldots, H_{n}\right\}$

$$
\frac{\operatorname{Max}_{\phi, k}\left\{h_{\mathrm{amb}}[H(\phi, k)]\right\}}{\operatorname{Min}_{\phi, k}\left\{h_{\mathrm{amb}}[H(\phi, k)]\right\}} \stackrel{!}{=} \operatorname{Min}
$$

where the function $H(\phi, k) \in\left\{H_{1}, \ldots, H_{n}\right\}$ represents the selected HELIX formation as function of latitude $\phi$ and swath number $k$. Note that the number of swaths depends on the geographic latitude since fewer swaths will be required for full coverage at higher latitudes. The previous optimization is then repeated for all sets of HELIX formations, and the set of HELIX formations yielding the lowest variation of the ambiguous height is regarded as optimum for DEM acquisition. For TanDEM-X, the set of possible HELIX formations is further restricted by the following constraints.

1) The cross-track separation between the satellite orbits shall always be larger than $150 \mathrm{~m}$. This minimum separation is required for safe operation. The libration phase $\psi$ should furthermore be kept in the interval from $150^{\circ}$ to $210^{\circ}$, which leaves at least a 17 -day safety margin until the eccentricity vectors coincide due to their natural rotation (see Section II-D).

2) The height of ambiguity should always be smaller than the maximum height of ambiguity meeting the

\footnotetext{
${ }^{7}$ Baseline stability means, of course, a reconstructed baseline based on a differential orbit model.
} 


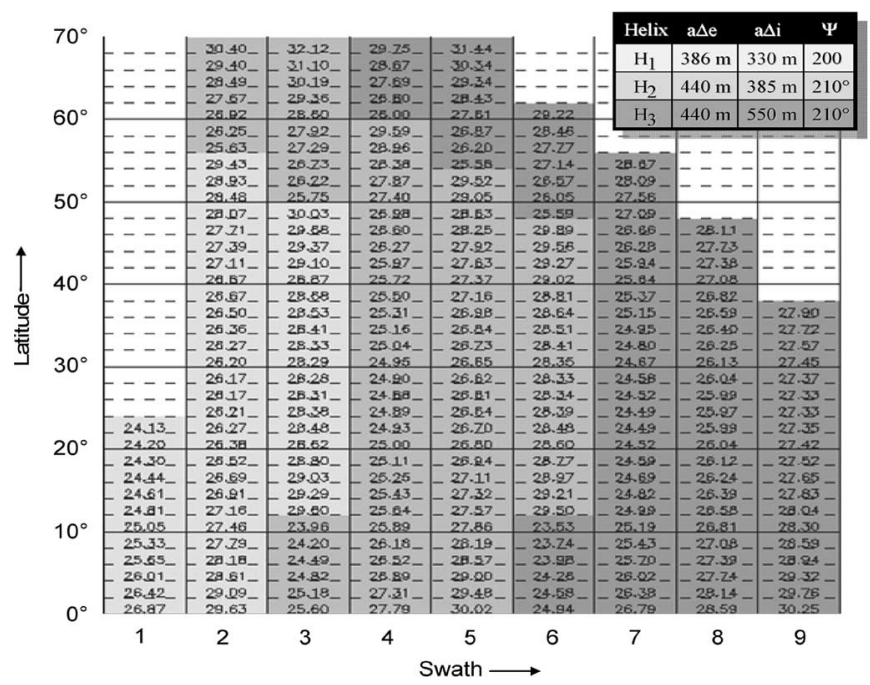

Fig. 20. (Right) HELIX parameters for DEM acquisition and (left) corresponding ambiguous heights as a function of the (horizontal) swath number and (vertical) latitude (in $2^{\circ}$ steps). The gray scales correspond to (light gray) $H_{1}$, (gray) $H_{2}$, and (dark gray) $H_{3}$.

HRTI-3 DEM performance requirement as outlined in Section III-C. This ensures that the relative height accuracy is better than $2 \mathrm{~m}$ for all latitudes within the considered incident angle range.

3) The resulting HELIX formations shall not require too much fuel for formation keeping. As outlined in Section II-D, this is tantamount to the requirement of minimizing the radial displacement $a \cdot \Delta e$ between the two satellites.

4) Each change of the HELIX formation will cost additional fuel and reconfiguration time. The number of HELIX formations should hence be minimized. A low number of HELIX formations will moreover increase the average time within each mission phase, thereby providing more flexibility for the scheduling of the individual data takes.

Fig. 20 shows the HELIX parameters and the corresponding ambiguous heights which have been derived from this optimization procedure for a latitude interval ranging from $\phi=-62^{\circ}$ up to $\phi=+70^{\circ}$. The higher northern latitudes and Antarctica will require an increased vertical displacement $a \cdot \Delta e$, and a dedicated formation has been derived independently in mapping the polar regions. This formation will not use a fixed libration phase, but we will allow for a natural rotation of the eccentricity vector. Daily orbit maintenance using the cold gas system can then be substituted by less frequent maneuvers using the more efficient hydrazine thrusters. The drift of the eccentricity vector during this mission phase is furthermore well suited to fill in the potential gaps remaining from the previous data acquisitions with an adequate height of ambiguity.

The gray areas in Fig. 20 show a variation of the height of ambiguity between 23.53 and $32.12 \mathrm{~m}$ which corresponds to a ratio of $\operatorname{Max}\left(h_{\mathrm{amb}}\right) / \operatorname{Min}\left(h_{\mathrm{amb}}\right)=1.36$. A height of ambiguity below $30 \mathrm{~m}$ is likely to cause phase-unwrapping problems for at least some regions with difficult terrain. To avoid such difficulties, we will map each point of the Earth landmass with a second increased height of ambiguity. This is in accordance with the system resources and the data rate constraints discussed in Section III-A. A combination of both acquisitions will facilitate phase unwrapping while still meeting the height accuracy requirements [48]. In principle, one should employ for the second data acquisition rather small baselines but still large enough that the height errors from this second acquisition remain well below the height of ambiguity from the first acquisition. However, this will not be feasible with TanDEM-X, since the choice of very short baselines will violate the minimum distance constraint previously introduced. We will hence use another technique where both interferograms are demodulated with respect to each other. In this way, we obtain a third interferogram with a height of ambiguity

$$
h_{\mathrm{amb}}=\frac{h_{\mathrm{amb}, 1} \cdot h_{\mathrm{amb}, 2}}{h_{\mathrm{amb}, 2}-h_{\mathrm{amb}, 1}}
$$

where $h_{\mathrm{amb}, 1}$ and $h_{\mathrm{amb}, 2}$ are the ambiguous heights from the first and the second data acquisition, respectively. In order to achieve again a homogeneous performance, we may simply scale the HELIX parameters $a \cdot \Delta e$ and $a \cdot \Delta i$ in Fig. 20 by a constant factor $\mu$. This ensures, for each point on the Earth surface, two interferometric acquisitions with an almost constant baseline ratio. The resulting height of ambiguity is then given by

$$
h_{\mathrm{amb}}=\frac{1}{1-\mu} \cdot h_{\mathrm{amb}, 1} .
$$

A scaling factor of $\mu=0.75$ will hence increase the differential height of ambiguity by a factor of four from, e.g., $h_{\mathrm{amb}, 1}=25 \mathrm{~m}$ to $h_{\mathrm{amb}}=100 \mathrm{~m}$. In principle, one could employ a factor $\mu$ which is even closer to one, thereby increasing the height of ambiguity further. The limit is then given by the accuracy of the formation control and the height accuracy. The use of two acquisitions with similar baseline lengths has the additional advantage of improving the relative height accuracy, as shown in Section III-C.

Remaining problems due to foreshortening, shadowing, and layover effects will be resolved by means of additional data takes after the completion of the second global DEM acquisition. The involved areas are adaptively identified from the previously recorded data. The additional acquisitions employ different incident angles and/or a combination of data takes from ascending and descending orbits. The latter requires either a roll maneuver of both satellites before each data take to acquire SAR images in the left-looking mode or a shift of the libration phase of TDX in the HELIX formation. A $180^{\circ}$ shift of the libration phase $\psi$ will be performed during the third mission year in acquiring the calibration data takes required for the bundle block adjustment. Residual height ambiguities can then be resolved by employing the maximum-likelihood technique suggested in [49].

\section{NeW IMAging TeChNiques}

The TanDEM-X mission will provide the remote sensing scientific community with a unique data set to exploit the capability of new bistatic radar techniques and to apply these innovative techniques for enhanced geo- and biophysical 
parameter retrievals. The following sections give a short overview of some of these potentials.

\section{A. Along-Track Interferometry}

TanDEM-X is predestinated for ATI, which compares the phase of two complex SAR images acquired in identical geometries but separated by a short time interval [50]-[52]. This technique is well suited in monitoring dynamic processes on the Earth's surface. As outlined in Section II, it is possible to adjust the along-track displacement between the two satellites from almost zero to several kilometers. Therefore, it becomes possible to adapt the ATI sensitivity of TanDEM-X to a wide range of radial velocities. Rather short baselines on the order of 50 to $100 \mathrm{~m}$ are, for example, desired for the measurement of ocean surface currents. For this, we note first that the signal returns from ocean surfaces decorrelate within very short time periods, the length of which depends on the actual wind speed. The decrease in coherence can be modeled by

$$
\gamma_{\text {Temp }}=\exp \left(-\frac{t^{2}}{\tau_{e}^{2}}\right)=\exp \left(-\frac{B_{\text {along }}^{2}}{4 \cdot \nu^{2} \cdot \tau_{e}^{2}}\right)
$$

where $\tau_{e}$ is the time lag leading to a $1 / e$ decay of the autocorrelation function. The factor four in the denominator on the right-hand side is due the assumed operation in bistatic mode where the effective along-track baseline is only half the actual distance between the two satellites (cf., Section II-A). The correlation model in (47) is in good agreement with the $\mathrm{X}$-band VV decorrelation functions shown in [53] if we use $\tau_{e}=\{4,6,12 \mathrm{~ms}\}$ for wind speeds of $\{15,10,5 \mathrm{~m} / \mathrm{s}\}$. Using (47), the interferometric phase errors are then computed according to Section III, where we assume an incident angle of $\theta_{\mathrm{i}}=40^{\circ}$. The scattering coefficients have been derived from [54] which yield for VV-polarization normalized radar crosssections of NRCS $=\{-12.1,-15.6,-21.5 \mathrm{~dB}\}$ for the three aforementioned wind speeds under upwind conditions. The accuracy of the velocity estimates in the ground range direction is finally approximated by [53]

$$
\sigma_{\nu}=\frac{\lambda \nu_{\text {sat }}}{2 \pi B_{\text {along }} \sin \theta_{\mathrm{i}}} \sigma_{\varphi}
$$

Fig. 21 shows the predicted accuracy for the three wind speeds as a function of the along-track separation between TSX and TDX. This example is based on a horizontal resolution of $50 \mathrm{~m} \times 50 \mathrm{~m}$. It becomes clear that the optimum along-track separation ranges from 35 to $150 \mathrm{~m}$, depending on the actual wind speed, while the along-track baselines above $150 \mathrm{~m}$ are likely to cause significant decorrelation in case of medium to high wind speeds. Very short along-track baselines below $100 \mathrm{~m}$ can be adjusted without any collision risk, owing to the HELIX formation.

The ATI can furthermore be performed by the so-called dualreceive antenna mode in each of the two tandem satellites, which provide each an additional along-track baseline of $2.4 \mathrm{~m}$ [55], [56]. TanDEM-X can hence be operated as an along-track SAR interferometer with four phase centers (cf., Fig. 22). The

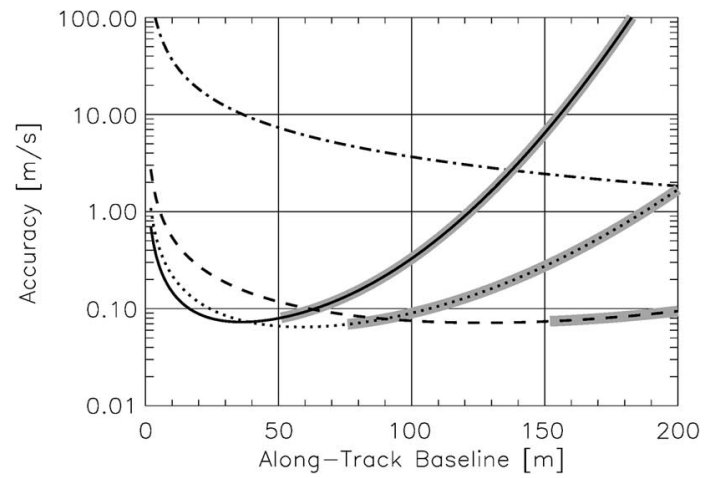

Fig. 21. Predicted accuracy of ocean current measurements for wind speeds of (dashed) 5 , (dotted) 10 , and (solid) $15 \mathrm{~m} / \mathrm{s}$ as a function of the along-track baseline. The gray tubes indicate baselines where the temporal correlation is lower than $\gamma_{\text {Temp }}=0.5$. For reference, the dashed-dotted curve shows also the ambiguous velocity.

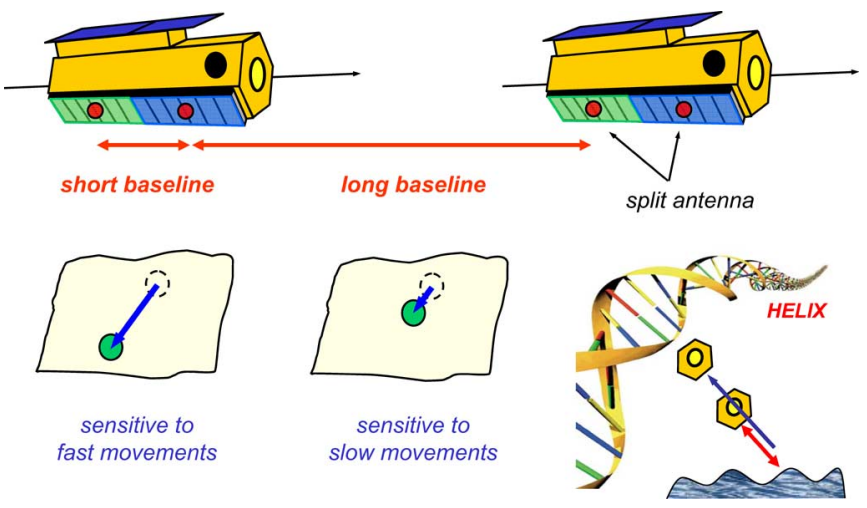

Fig. 22. ATI with TanDEM-X. The HELIX orbit concept allows a flexible adjustment of the desired along-track separation between the satellites. In addition, a short along-track baseline is provided by each satellite.

combination of short and long baseline ATI data acquisitions can then, for example, be used to resolve phase ambiguities from high-velocity scatterers. The advantage of an additional short baseline becomes also evident from Fig. 21, where the upper dashed-dotted curve shows the $2 \pi$ ambiguous velocity which has a value of $5 \mathrm{~m} / \mathrm{s}$ for an along-track baseline of $75 \mathrm{~m}$. For comparison, the accuracy with the split antenna mode in TSX and TDX will be 1-2 $\mathrm{m} / \mathrm{s}$, which is sufficient to resolve the phase ambiguity from the large baseline measurement.

A further potential arises from the alternating bistatic mode which enables the acquisition of two effective along-track baselines separated by a factor of two in a single pass [57]. This will not only improve the interferometric performance by an adaptive selection of the time lag for the different wind speeds but it also enables a direct measurement and compensation of the additional Doppler shift induced by the Bragg scattering mechanism, thereby avoiding, e.g., the need for ancillary wind information for ocean current retrieval [58]. Vice versa, this may also open a new opportunity for wind speed measurements.

Another potential of ATI with TanDEM-X is the monitoring of moving objects on the ground. The employment 
of this technique from spaceborne sensors recently received much interest with regard to applications like wide-area traffic monitoring, ship detection, and ground surveillance [59]-[62]. The feasibility of spaceborne traffic monitoring was first demonstrated during the SRTM mission where an evaluation of the interferometric phase enabled the detection of large vehicles [63]. The cross-track velocity was then indirectly derived from the azimuth displacement by exploiting the train-off-the-track effect which requires the availability of an accurate road database [59]. TanDEM-X has the potential to significantly improve the detection, velocity estimation, and localization of moving objects by exploiting, in addition, the along-track baseline between the two satellites. An along-track baseline of $50 \mathrm{~m}$ yields for $\theta_{\mathrm{i}}=40^{\circ}$ a cross-track sensitivity of $0.05 \mathrm{~km} / \mathrm{h} / \mathrm{deg}$. By noting that a car with a RCS of $0 \mathrm{dBm}^{2}$ yields a phase error below $10^{\circ}$, one may arrive at velocity accuracies on the order of $1 \mathrm{~km} / \mathrm{h}$, depending on the actual along-track baseline. The feasibility of ground-moving target indication in a bistatic airborne SAR configuration has already been demonstrated in [64]. The HELIX formation allows not only for an adaptation of the along-track separation with regard to the specific application, but it is also possible to simultaneously minimize the across-track component for a given latitude and incident angle range, as shown in the lower right of Fig. 22. This is important for demonstration purposes, since it significantly alleviates the processing by reducing the phase contamination from topography. Even more information about the moving object and its direction could, in principle, be obtained by employing the simultaneous transmit mode $^{8}$ which enables the acquisition of coherent information from pointlike targets even in case of nonoverlapping frequency bands (cf., [65]). The availability of multiple baselines will then help to derive highly accurate velocity and position estimates without explicit recourse to a road database [66].

The ATI mode is, moreover, of high value for coherence analyses in the vegetated areas, where decorrelation times below $100 \mathrm{~ms}$ have been observed in the X-band in case of moderate to strong wind conditions [38]. By employing the alternating bistatic mode, one will obtain accurate estimates of the decorrelation times for the vegetated areas in a way that is similar to the multibaseline coherence time measurements for oceanographic mapping [57]. The coherence maps may then be used to improve land cover classification and/or to get firstorder wind speed estimates. A further promising application to be demonstrated with TanDEM-X is the monitoring of sea ice drift which will presumably require large baselines in combination with an operation in the pursuit monostatic mode. The along-track baselines of several kilometers will be available in the beginning and at the end of the mission. The large alongtrack baselines could also be of benefit for traffic monitoring, since they will enable an estimation of the velocity vector from

\footnotetext{
${ }^{8}$ The employment of the alternating transmit mode has the disadvantage of reducing the effective PRF for each channel. A high PRF on the order of $6 \mathrm{kHz}$ is, however, desired for the unambiguous azimuth focusing of fast vehicles.
}

the relative displacement of the moving objects in the two SAR images, while the detection could be based on the split antennas in each satellite.

\section{B. Very Large Baseline Cross-Track Interferometry}

Very large baseline interferometry takes advantage of the high RF bandwidth of the TSX and TDX satellites which allows for coherent data acquisitions with baselines of up to $5 \mathrm{~km}$ and more. Note in this context that less than $5 \%$ of the maximum possible (critical) baseline length is used during the nominal DEM data acquisition (cf., Fig. 15) which is, furthermore, based on a reduced chirp bandwidth to limit the overall data rate as required for global multibaseline coverage. Large baseline interferograms with higher bandwidth can hence significantly improve the height accuracy for local areas, but the associated low height of ambiguity requires a combination of multiple interferograms with different baseline lengths to resolve phase ambiguities [49], [67], [68]. By using this technique, it becomes possible to derive DEMs with HRTI-4-like accuracy on a local or even regional scale.

Further opportunities arise from the comparison of multiple very large baseline TanDEM-X interferograms acquired during different formation passes. This provides a sensitive measure for vertical scene and structure changes. Potential applications are a detection of the grounding line which separates the shelf from the inland ice in polar regions, monitoring of vegetation growth, mapping of atmospheric water vapor with high spatial resolution, measurement of snow accumulation, and detection of anthropogenic changes of the environment, e.g., due to deforestation. Note that most of these combinations rely on a comparison of two or more single-pass (large baseline) crosstrack interferograms and do not require coherence between the different passes (cf., Fig. 23).

Further information can be gained from an evaluation of coherence changes between different passes-potentially augmented by polarimetric information. This could, for instance, reveal even slight changes in the soil and vegetation structure, reflecting vegetation growth and loss, freezing and thawing, fire destruction, human activities, and so on. Another, and in some sense, complementary opportunity is the demonstration of a quasi-tomographic mapping of, for example, loose vegetation. For this, we note first that it is unlikely to obtain in the X-band a sufficient coherence between subsequent passes as required to employ classical tomography via a linear beamforming process [69]. An alternative is the combination of multiple single-pass SAR interferograms acquired with different baseline lengths. This enables then a reconstruction of the vertical layer structure by employing the Van Cittert Zernike theorem [cf., (29)]. Such a partially coherent tomographic mapping technique could also incorporate vertical structure functions, as suggested recently in [70] for polarization coherent tomography, thereby improving the reconstruction results in case of a limited number of single-pass SAR interferograms. The systematic combination of multiple single-pass SAR interferograms acquired by TanDEM-X is hence likely to pave the way to a new age of interferometric and tomographic processing techniques and applications as it 

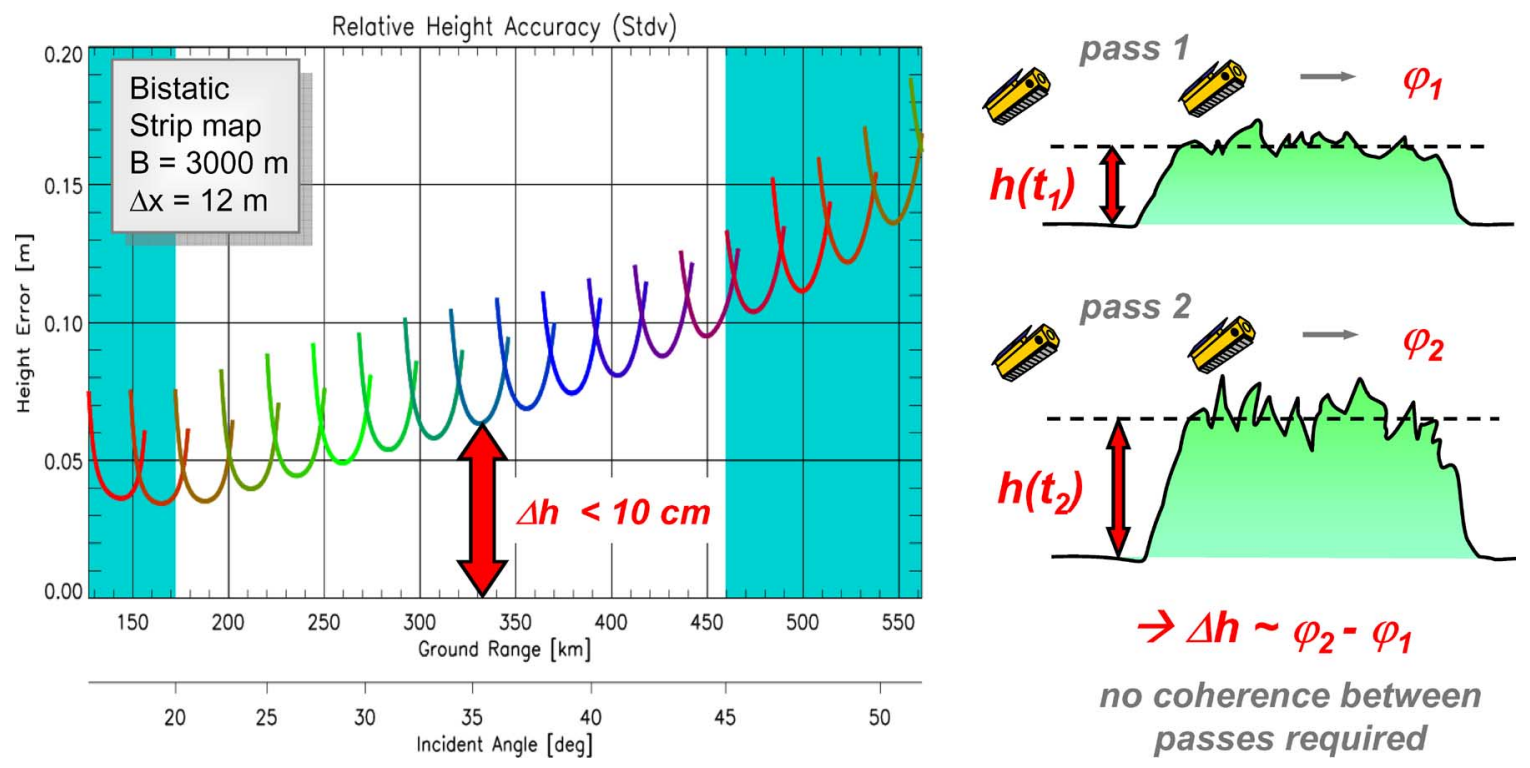

Fig. 23. Performance example for double differential SAR interferometry with TanDEM-X (baseline $=3 \mathrm{~km}$, posting $=12 \mathrm{~m}$ ). A relative height accuracy (standard deviation) better than $10 \mathrm{~cm}$ is achieved. This example uses the standard TerraSAR-X stripmap beams and a bandwidth of $150 \mathrm{MHz}$. The vertical resolution can be improved by increasing the postspacing.

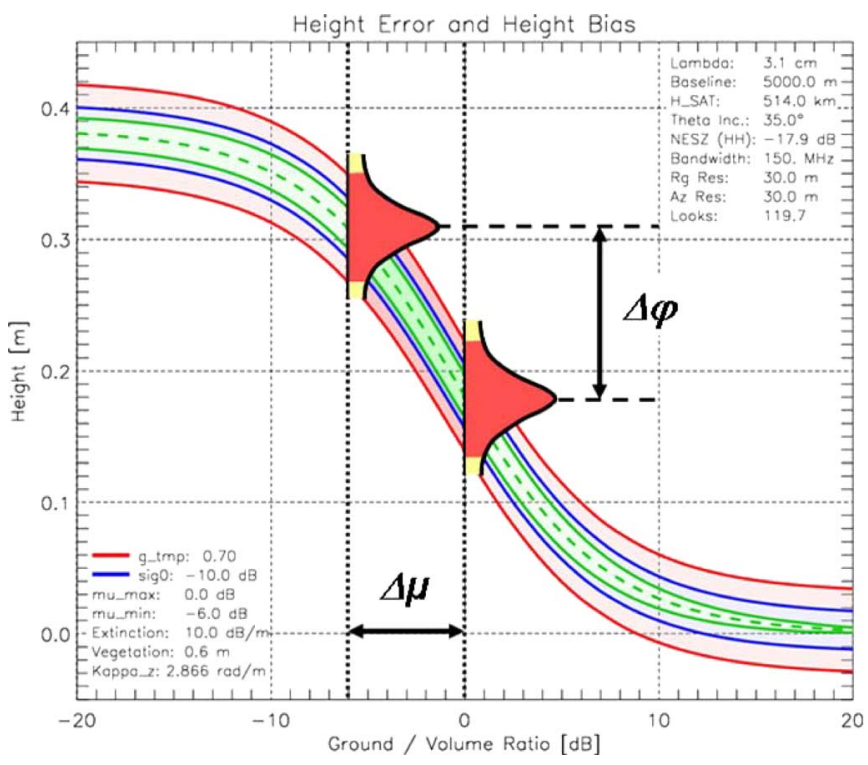

Fig. 24. Vertical separation of interferometric phase centers in TanDEM-X as a function of the ground-to-volume scattering ratios $\mu$ (cf., [74]). The vegetation height is $0.6 \mathrm{~m}$, the ground-to-volume ratio $\mu$ varies from -6 to $0 \mathrm{~dB}$, and the extinction is assumed to be $10 \mathrm{~dB} / \mathrm{m}$.

was ERS-1/2 for the development of classical repeat-pass SAR interferometry.

\section{Polarimetric SAR Interferometry}

Polarimetric SAR interferometry combines interferometric with polarimetric measurements to gain additional information from semitransparent volume scatterers [15], [71]. This allows, for example, the extraction of important biophysical parameters like vegetation density and vegetation height [72]. Fully polarimetric operation uses the split antenna and is susceptible to ambiguities similar to the alternating bistatic mode (cf., Fig. 3). This can be avoided by employing steep incident angles, reducing the processed azimuth bandwidth, and/or by limiting the swath width. Fig. 24 shows the achievable performance of a simulated scenario for TanDEM-X. This analysis is based on the Random Volume over Ground model [15], [71], [72] assuming a vegetation layer with a height of $0.6 \mathrm{~m}$ and an extinction coefficient of $10 \mathrm{~dB} / \mathrm{m}$, which are typical values in an agriculture scenario [73]. The dashed line indicates the height variation of the interferometric phase center with different polarizations (corresponding to a variation of $\mu$ on the abscissa). The inner tube shows the height errors due to volume decorrelation for an effective baseline of $5 \mathrm{~km}$ and an independent postspacing of $30 \mathrm{~m} \times 30 \mathrm{~m}$. The middle tube shows additional errors due to the limited system accuracy, and the outer tube indicates potential errors in case of temporal decorrelation caused by a possible along-track separation between the two satellites (here, $\gamma_{\text {Temp }}=0.7$ ). The performance analysis predicts, for this example, a sufficient vertical phase center separation to enable a successful retrieval of important vegetation parameters like volume height, extinction, etc. Note in this context that there may be a significant difference between the vertical coherence loci of mono- and bistatic SAR interferograms if the ground returns are dominated by a double-bounce scattering mechanism [16]. TanDEM-X offers the opportunity to acquire interferograms in both the bistatic and the pursuit monostatic mode, and a comparison of both types of interferograms provides a valuable insight into the dominant ground scattering process. Such a comparison could, in principle, also be based on the alternating transmit mode, but in this case, one will be restricted to one co- and one cross-polarized component since the SAR ambiguities prevent a simultaneous operation in fully polarimetric and alternating bistatic mode. Another opportunity to be investigated with TanDEM-X is the use of the simultaneous transmit mode in combination with a large interferometric baseline as required for low vegetation. This will allow for the acquisition of fully polarized inter- 
ferometric data with two baselines. TanDEM-X will be the first mission to demonstrate such innovative techniques from space.

\section{Bistatic SAR Imaging}

Bistatic SAR imaging provides additional observables for the extraction of important scene and target parameters [75], [76]. TanDEM-X allows for the simultaneous acquisition of bistatic and monostatic SAR images in a single data take to obtain a highly informative set of multiangle observations. Note that it is possible to collect up to two different monostatic and bistatic SAR images during a single satellite formation pass by employing either the alternating bistatic or the simultaneous transmit mode. The acquisition of two bistatic SAR images in identical geometry but with mutually interchanged transmitters will also be of high interest for polarimetric analyses due to the asymmetry between the cross-polarized channels. A quantitative evaluation of the bistatic RCS and a comparison with its monostatic equivalents furthermore facilitate the detection and recognition of targets. The segmentation and classification in radar images are expected to be substantially improved by comparing the spatial statistics of mono- and bistatic scattering coefficients. This is also supported by the joint airborne bistatic radar experiments performed by DLR and ONERA, which revealed significant changes of the scattering behavior for both artificial and natural targets even in case of rather small bistatic angles [77]. A joint evaluation of mono- and bistatic SAR images could furthermore be used to isolate different scattering mechanisms, like, for example, a distinction between highly directive dihedral returns from more isotropic volume scattering. The bistatic SAR imaging moreover has a potential for the retrieval of sea state parameters, the estimation of surface roughness and terrain slope, as well as stereogrammetric, meteorological, and atmospheric applications [75]. Innovative processing algorithms will be required to exploit all these capabilities. The bistatic data acquired with TanDEM-X will hence provide a unique data source to improve our understanding of bistatic imaging and its exploitation for future remote-sensing applications. Data takes with large bistatic angles are planned at the beginning and at the end of the TanDEM-X mission where the satellites are separated from each other by several tenths of kilometers.

\section{E. Superresolution and Digital Beamforming}

Another promising technique is superresolution [78]. This technique exploits the fact that the signals received by the two satellites have different aspect angles for each scattering point on the ground. In consequence, the two ground range and/or azimuth spectra are shifted relative to each other. A coherent combination of the signals yields then a wider spectrum which corresponds to an improved spatial resolution. This technique requires a cross-track and/or along-track separation on the order of 5 to $10 \mathrm{~km}$. The large cross-track baselines will be available in later mission phases after the standard DEM data acquisition has been completed. The application of the superresolution technique in range may furthermore take advantage of the al- ternating bistatic mode, where the bistatic interferograms serve as a "phase bridge" to link two nonoverlapping monostatic SAR spectra.

A complementary technique to superresolution is digital beamforming on receive which combines the RF signals from multiple antennas to simulate a large directional antenna. Due to the split antennas and dual receiver channels of TSX and TDX, up to four phase centers can be obtained in the standard bistatic stripmap mode. A combination of the multiple $\mathrm{Rx}$ signals enables then an efficient suppression of azimuth ambiguities [79]. In this way, it becomes possible to demonstrate the capabilities of high-resolution wide-swath SAR imaging [80]. Digital beamforming on receive is also of interest for advanced interferometric SAR modes like the alternating bistatic mode where it allows for a reduction of the PRF, thereby resolving potential timing and ambiguity conflicts. While it is straightforward to combine the recorded signals from the split antenna in each of the two receiver satellites independently [79], new algorithms will be required to combine the signals from the two satellites for advanced azimuth ambiguity suppression in case of a nonvanishing cross-track separation between the platforms. The latter requires a compensation of the topographic phase and will lead to a new class of SAR processing techniques which combine the cross-track interferometry with the alongtrack digital beamforming [76]. TanDEM-X will be the first configuration to demonstrate this highly innovative technique from space.

\section{CONCLUSION}

This paper demonstrated the capability of TanDEM-X to acquire a global DEM in accordance with the HRTI-3 standard. The achievable height accuracy for global DEM generation is, in practice, mainly limited by the height of ambiguity that can finally be processed during phase unwrapping. The standard HRTI-3 DEM is a reasonable compromise between performance, processing, and data acquisition effort. A mission scenario has been developed which enables the acquisition of a global HRTI-3 DEM within less than three years. This concept includes several data takes with different baselines, different incident angles, and data takes from ascending and descending orbits to deal with difficult terrains, like mountains, valleys, tall vegetation, etc. The TanDEM-X mission concept allocates also sufficient acquisition time and satellite resources to secondary mission goals like ATI or the demonstration of new bistatic radar techniques.

In 2006, the TanDEM-X mission has been approved for realization by means of a PPP between the DLR and the EADS Astrium GmbH. The launch of the TanDEM-X satellite is planned for summer 2009 which ensures at least three years of joint operation with the TSX. Current work includes the preparation of a robust data acquisition plan, which has also to take into account potential conflicts between the TerraSAR-X and the TanDEM-X mission objectives, the development of an advanced DEM calibration concept, the design of a multibaseline InSAR processor, performance investigations for the other innovative TanDEM-X imaging modes, as well as the compilation of a detailed science plan. 


\section{APPENDIX}

Formula (36) yields a good approximation for TanDEM-X in conjunction with the independent zero Doppler steering. In the general case of different antenna patterns, it may, however, underestimate the effective number of looks. This is particularly pronounced if the spectral weightings of the two interferometric channels significantly differ from each other, thereby reducing also the coherence [cf., (27)]. The asymmetric spectral weighting introduces systematic phase fluctuations in the interferogram which become the dominating error in case of a high SNR where the superposition from the receiver noise with its white power spectral density can be neglected. The smoothing of the interferogram via multilooking will then remove this systematic component with more efficiency than expected from a mere superposition with independent white noise. In consequence, the effective number of looks is no longer independent from the actual SNR. Following the reasoning in [43], a good approximation of the effective number of looks can be obtained by comparing the error variances before and after the final smoothing filter. The errors in our case can be approximated by the power spectral densities of the interferogram's imaginary part where we assume, for convenience, zero topographic phase. After some tedious algebra, the correlation ratio can be derived as

$$
\begin{aligned}
n_{\mathrm{az}}=\iint H\left(f_{1}, f_{2}\right) \cdot d f_{1} \cdot d f_{2} / \iint H\left(f_{1}, f_{2}\right) \\
\cdot H_{\text {smooth }}^{2}\left(f_{2}-f_{1}\right) \cdot d f_{1} \cdot d f_{2}
\end{aligned}
$$

where

$$
\begin{gathered}
H\left(f_{1}, f_{2}\right)=H_{\text {proc }}^{2}\left(f_{1}\right) \cdot H_{\text {proc }}^{2}\left(f_{2}\right) \\
\cdot\left\{\mathrm{SNR}^{2}\left[H_{a}\left(f_{1}\right) H_{b}\left(f_{2}\right)-H_{a}\left(f_{2}\right) H_{b}\left(f_{1}\right)\right]^{2}\right. \\
+\operatorname{SNR}\left[H_{a}^{2}\left(f_{1}\right)+H_{a}^{2}\left(f_{2}\right)+H_{b}^{2}\left(f_{1}\right)\right. \\
\left.\left.+H_{b}^{2}\left(f_{2}\right)\right]+2\right\}
\end{gathered}
$$

with $\quad H_{a}(f)=A_{\mathrm{Tx}}(f) \cdot A_{\mathrm{Rx} 1}(f) \quad$ and $\quad H_{b}(f)=A_{\mathrm{Tx}}(f)$. $A_{\mathrm{Rx} 2}(f)$. Formula (36) approximates this equation for $H_{a}(f)=H_{b}(f)$ and SNR $\gg 1$. Computer simulations with various antenna weightings and smoothing filters show that the previous equations yield, in combination with (31), good predictions for both the $90 \%$ errors and the standard deviations. One has, however, also to be aware that the multilook phase error pdf in (31) is, in a strict sense, no longer valid, since the interferometric phase error pdf becomes slightly different in shape, which is a fact that cannot be corrected for by a mere adjustment of the number of looks.

\section{ACKNOWLEDGMENT}

The authors would like to thank the anonymous reviewers for their valuable comments and suggestions that helped to improve this paper. The authors would also like to thank all members of the joint DLR-EADS Astrium Team for their continuous effort and enthusiasm to implement the ambitious TanDEM-X mission in space as well as the numerous members of the TanDEM-X Science Team for their inputs and comments.

\section{REFERENCES}

[1] H. A. Zebker, T. G. Farr, R. P. Salazar, and T. H. Dixon, "Mapping the world's topography using radar interferometry: The TOPSAT mission," Proc. IEEE, vol. 82, no. 12, pp. 1774-1786, Dec. 1994.

[2] A. Moreira, G. Krieger, I. Hajnsek, M. Werner, D. Hounam, S. Riegger, and E. Settelmeyer, "TanDEM-X: A TerraSAR-X add-on satellite for single-pass SAR interferometry," in Proc. IGARSS, Anchorage, AK, 2004, pp. 1000-1003.

[3] I. Hajnsek and A. Moreira, "TanDEM-X: Mission and science exploration," in Proc. EUSAR, Dresden, Germany, May 2006.

[4] N. El-Sheimy, C. Valeo, and A. Habib, Digital Terrain Modeling: Acquisition, Manipulation, and Applications. London, U.K.: Artech House, 2005.

[5] Z. Li, Q. Zhu, and C. Gold, Digital Terrain Modeling. New York: CRC Press, 2005

[6] P. A. Rosen, S. Hensley, I. R. Joughin, F. K. Li, S. N. Madsen, E. Rodriguez, and R. Goldstein, "Synthetic aperture radar interferometry," Proc. IEEE, vol. 88, no. 3, pp. 333-382, Mar. 2000.

[7] M. Werner, "Shuttle Radar Topography Mission (SRTM): Mission overview," J. Telecommun. (Frequenz), vol. 55, no. 3/4, pp. 75-79, 2001.

[8] B. Rabus, M. Eineder, A. Roth, and R. Bamler, "The Shuttle Radar Topography Mission-A new class of digital elevation models acquired by spaceborne radar," ISPRS J. Photogramm. Remote Sens., vol. 57, no. 4, pp. 241-262, Feb. 2003.

[9] TanDEM-X WEB page. [Online]. Available: http://www.dlr.de/hr/tdmx

[10] National Imagery and Mapping Agency (NIMA), High Resolution Terrain Information (HRTI): Performance Specification, 2000. MIL-PRF-89048.

[11] R. Hanssen, Radar Interferometry. Dordrecht, The Netherlands: Kluwer, 2001.

[12] M. Stangl, R. Werninghaus, B. Schweizer, C. Fischer, M. Brandfass, J. Mittermayer, and H. Breit, "TerraSAR-X technologies and first results," Proc. Inst. Electr. Eng.-Radar, Sonar Navig., vol. 153, no. 2, pp. 86-95, Apr. 2006.

[13] A. Moccia, G. Salzillo, M. D'Errico, G. Rufino, and G. Alberti, "Performance of spaceborne bistatic synthetic aperture radar," IEEE Trans. Aerosp. Electron. Syst., vol. 41, no. 4, pp. 1383-1395, Oct. 2005.

[14] H. Fiedler, E. Boerner, J. Mittermayer, and G. Krieger, "Total zero Doppler steering-A new method for minimizing the Doppler centroid," IEEE Geosci. Remote Sens. Lett., vol. 2, no. 2, pp. 141-145, Apr. 2005.

[15] R. N. Treuhaft and P. R. Siqueira, "The vertical structure of vegetated land surfaces from interferometric and polarimetric radar," Radio Sci., vol. 35, no. 1, pp. 141-177, 2000 .

[16] J. D. Ballester-Berman and J. M. Lopez-Sanchez, "Coherence loci for a homogeneous volume over a double bounce ground model," IEEE Geosci. Remote Sens. Lett., vol. 4, no. 2, pp. 317-321, Apr. 2007.

[17] G. Krieger and A. Moreira, Spaceborne Interferometric and Multi-Static SAR Systems. Hoboken, NJ: Wiley, 2007. to appear in M. Cherniakov: Bistatic Radar: Emerging Technology.

[18] F. Gatelli, A. M. Guamieri, F. Parizzi, P. Pasquali, C. Prati, and F. Rocca, "The wavenumber shift in SAR interferometry," IEEE Trans. Geosci. Remote Sens., vol. 32, no. 4, pp. 855-865, Jul. 1994.

[19] G. Krieger and M. Younis, "Impact of oscillator noise in bistatic and multistatic SAR," IEEE Geosci. Remote Sens. Lett., vol. 3, no. 3, pp. 424428, Jul. 2006.

[20] R. Bamler and P. Hartl, "Synthetic aperture radar interferometry," Inv. Probl., vol. 14, no. 4, pp. 1-54, Aug. 1998.

[21] H. Braubach and M. Völker, "Method for drift compensation with radar measurements with the aid of reference radar signals," U.S. Patent 2005/0 083225 A1, U.S. Off. Pat. Office, Washington, DC, Apr. 21, 2005.

[22] J. Rutman, "Characterization of phase and frequency instabilities in precision frequency sources: Fifteen years of progress," Proc. IEEE, vol. 66, no. 9, pp. 1048-1075, Sep. 1978.

[23] M. Younis, R. Metzig, and G. Krieger, "Performance prediction of a phase synchronization link for bistatic SAR," IEEE Geosci. Remote Sens. Lett., vol. 3, no. 3, pp. 429-433, Jul. 2006.

[24] A. Moreira, G. Krieger, and J. Mittermayer, "Satellite configuration for interferometric and/or tomographic remote sensing by means of synthetic aperture radar (SAR)," U.S. Patent 6677 884, U.S. Off. Pat. Office, Washington, DC, Jul. 2002.

[25] H. Fiedler and G. Krieger, "Close formation of passive receiving microsatellites," in Proc. 18th Int. Symp. Space Flight Dyn., Munich, Germany, 2004, pp. 47-52. 
[26] S. D'Amico, O. Montenbruck, C. Arbinger, and H. Fiedler, "Formation flying concept for close remote sensing satellites," in Proc. 15th AAS/AIAA Space Flight Mech. Conf., Copper Mountain, CO, 2005, pp. 831-848.

[27] D. Brouwer and G. M. Clemence, Methods of Celestial Mechanics. New York: Academic, 1961.

[28] G. W. Hill, "Researches in the lunar theory," Amer. J. Math., vol. 1, no. 1, pp. 5-26, 1878.

[29] W. H. Clohessy and R. S. Wiltshire, "Terminal guidance systems for satellite rendezvous," J. Aerosp. Sci., vol. 270, no. 9, pp. 653-658, 1960.

[30] F. T. Ulaby and M. C. Dobson, Handbook of Radar Scattering Statistics for Terrain. Norwood, MA: Artech House, 1989.

[31] E. Rodriguez and J. M. Martin, "Theory and design of interferometric synthetic aperture radars," Proc. Inst. Electr. Eng. F-Radar Signal Process., vol. 139, no. 2, pp. 147-159, Apr. 1992

[32] H. A. Zebker and J. Villasenor, "Decorrelation in interferometric radar echoes," IEEE Trans. Geosci. Remote Sens., vol. 30, no. 5, pp. 950-959, Sep. 1992.

[33] D. Just and R. Bamler, "Phase statistics of interferograms with applications to synthetic aperture radar," Appl. Opt., vol. 33, no. 20, pp. 43614368, Jul. 1994.

[34] J. C. Curlander and R. N. McDonough, Synthetic Aperture Radar: Systems and Signal Processing. New York: Wiley, 1991.

[35] I. H. McLeod, I. G. Cumming, and M. S. Seymour, "ENVISAT ASAR data reduction: Impact on SAR interferometry," IEEE Trans. Geosci. Remote Sens., vol. 36, no. 2, pp. 589-602, Mar. 1998.

[36] N. S. Jayant and P. Noll, Digital Coding of Waveforms. London, U.K.: Prentice-Hall, 1984

[37] F. K. Li and R. M. Goldstein, "Studies of multibaseline spaceborne interferometric synthetic aperture radars," IEEE Trans. Geosci. Remote Sens., vol. 28, no. 1, pp. 88-97, Jan. 1990.

[38] R. M. Narayanan, D. W. Doerr, and D. C. Rundquist, "Temporal decorrelation of X-band backscatter from wind-influenced vegetation," IEEE Trans. Aerosp. Electron. Syst., vol. 28, no. 2, pp. 404-412, Apr. 1992.

[39] J.-S. Lee, K. W. Hoppel, S. A. Mango, and A. R. Miller, "Intensity and phase statistics of multilook polarimetric and interferometric SAR imagery," IEEE Trans. Geosci. Remote Sens., vol. 32, no. 5, pp. 10171028, Sep. 1994.

[40] M. Abramowitz and I. Stagun, Handbook of Mathematical Functions. New York: Dover, 1965

[41] A. Papoulis, Probability, Random Variables, and Stochastic Processes. New York: McGraw-Hill, 1991.

[42] L. R. Joughin and D. P. Winebrenner, "Effective number of looks for a multilook interferometric phase distribution," in Proc. IEEE Geosci. Remote Sens. Symp., Pasadena, CA, 1994, pp. 2276-2278.

[43] H. J. Thiebaux and F. W. Zwiers, "The interpretation and estimation of effective sample size," J. Clim. Appl. Meteorol., vol. 23, no. 5, pp. 800811, May 1984.

[44] R. Kroes, O. Montenbruck, W. Bertiger, and P. Visser, "Precise GRACE baseline determination using GPS," GPS Solut., vol. 9, no. 1, pp. 21-31, Apr. 2005.

[45] A. Moccia and S. Vetrella, "A tethered interferometric synthetic aperture radar (SAR) for a topographic mission," IEEE Trans. Geosci. Remote Sens., vol. 30, no. 1, pp. 103-109, Jan. 1992.

[46] M. Bachmann, J. Hueso González, H. Fiedler, S. Huber, G. Krieger, and M. Zink, "TanDEM-X calibration-Error analysis and DEM adjustment," in Proc. Int. Conf. Radar Syst., Edinburgh, U.K., 2007.

[47] C. C. Carabajal and D. J. Harding, "ICESat validation of SRTM C-band digital elevation models," Geophys. Res. Lett., vol. 32, no. 22, pp. L22S01.1-L22S01.5, 2005.

[48] M. Eineder and G. Krieger, "Interferometric digital elevation model reconstruction-Experiences from SRTM and multi channel approaches for future missions," in Proc. IGARSS, Seoul, Korea, 2005, pp. 2664-2667.

[49] M. Eineder and N. Adam, "A maximum-likelihood estimator to simultaneously unwrap, geocode, and fuse SAR interferograms from different viewing geometries into one digital elevation model," IEEE Trans. Geosci. Remote Sens., vol. 43, no. 1, pp. 24-36, Jan. 2005.

[50] R. M. Goldstein and H. A. Zebker, "Interferometric radar measurement of ocean surface currents," Nature, vol. 328, no. 20, pp. 707-709, Aug. 1987.

[51] A. Moccia and G. Rufino, "Spaceborne along-track SAR interferometry: Performance analysis and mission scenarios," IEEE Trans. Aerosp. Electron. Syst., vol. 37, no. 1, pp. 199-213, Jan. 2001.

[52] R. Romeiser, M. Schwäbisch, J. Schulz-Stellenfleth, D. Thompson, R. Siegmund, A. Niedermeier, W. Alpers, and S. Lehner, Study of concepts for radar interferometry from satellites for ocean (and land) ap- plications. report. [Online]. Available: http://www.ifm.uni-hamburg.de/ $\sim$ Wwwrs/

[53] R. Romeiser and D. R. Thompson, "Numerical study on the along-track interferometric radar imaging mechanism of oceanic surface currents," IEEE Trans. Geosci. Remote Sens., vol. 38, no. 1, pp. 446-458, Jan. 2000.

[54] H. Masuko, K. Okamoto, M. Shimada, and S. Niwa, "Measurement of microwave backscattering signatures of the ocean surface using $\mathrm{X}$ band and Ka band airborne scatterometers," J. Geophys. Res., vol. 91, no. C11, pp. $13065-13083$, Nov. 1986.

[55] J. Mittermayer and H. Runge, "Conceptual studies for exploiting the TerraSAR-X dual receive antenna," in Proc. IGARSS, Toulouse, France, 2003, pp. 2140-2142.

[56] R. Romeiser and H. Runge, "Theoretical evaluation of several possible along-track InSAR modes of TerraSAR-X for ocean current measurements," IEEE Trans. Geosci. Remote Sens., vol. 45, no. 1, pp. 21-35, Jan. 2007.

[57] R. E. Carande, "Estimating ocean coherence time using dual-baseline interferometric synthetic aperture radar," IEEE Trans. Geosci. Remote Sens., vol. 32, no. 4, pp. 846-854, Jul. 1994

[58] F. Lombardini, F. Bordoni, F. Gini, and L. Verrazzani, "Multibaseline ATISAR for robust ocean surface velocity estimation," IEEE Trans. Aerosp. Electron. Syst., vol. 40, no. 2, pp. 417-433, Apr. 2004.

[59] F. Meyer, S. Hinz, R. Müller, G. Palubinskas, C. Laux, and H. Runge, "Towards traffic monitoring with TerraSAR-X," Can. J. Remote Sens., vol. 33, no. 1, pp. 39-51, 2007.

[60] K. H. Bethke, S. Baumgartner, M. Gabele, D. Hounam, E. Kemptner, D. Klement, G. Krieger, and R. Erxleben, "Air- and spaceborne monitoring of road traffic using SAR moving target indication-Project TRAMRAD," ISPRS J. Photogramm. Remote Sens., vol. 33, no. 1, pp. 39-51, Dec. 2006.

[61] C. Gierull and C. Livingstone, "SAR-GMTI concept for RADARSAT-2," in Applications of Space-Time Adaptive Processing, R. Klemm, Ed. London, U.K.: IEE, 2004, pp. 177-206.

[62] P. Lombardo, F. Colone, and D. Pastina, "Monitoring and surveillance potentialities obtained by splitting the antenna of the COSMO-SkyMed SAR into multiple sub-apertures," Proc. Inst. Electr. Eng.-Radar, Sonar Navig., vol. 153, no. 2, pp. 104-116, Apr. 2006.

[63] S. Suchandt, M. Eineder, H. Breit, and H. Runge, "Analysis of ground moving objects using SRTM/X-SAR data," ISPRS J. Photogramm. Remote Sens., vol. 61, no. 3/4, pp. 209-224, Dec. 2006.

[64] H. Cantalloube, P. Dubois-Fernandez, V. Giroux, and G. Krieger, "Bistatic moving target indication using across-track and along-track interferometry," in Proc. EUSAR, Dresden, Germany, 2006.

[65] R. Z. Schneider, K. P. Papathanassiou, I. Hajnsek, and A. Moreira, "Polarimetric and interferometric characterization of coherent scatterers in urban areas," IEEE Trans. Geosci. Remote Sens., vol. 44, no. 4, pp. 971-984, Apr. 2006.

[66] D. Cerutti-Maori and J. Ender, "Performance analysis of multistatic configurations for spaceborne GMTI based on the auxiliary beam approach," Proc. Inst. Electr. Eng.-Radar, Sonar Navig., vol. 153, no. 2, pp. 96-103, Apr. 2006.

[67] A. Ferretti, C. Monti Guarnieri, C. Prati, and F. Rocca, "Multibaseline interferometric techniques and applications," in Proc. Fringe Workshop, Zurich, Switzerland, 1996.

[68] G. Fornaro, C. Monti Guarnieri, A. Pauciullo, and S. Tebaldini, "Joint multi-baseline SAR interferometry," EURASIP J. Appl. Signal Process., vol. 20, pp. 3194-3205, 2005.

[69] A. Reigber and A. Moreira, "First demonstration of airborne SAR tomography using multibaseline L-band data," IEEE Trans. Geosci. Remote Sens., vol. 38, no. 5, pp. 2142-2152, Sep. 2000.

[70] S. Cloude, "Polarization coherence tomography," Radio Sci., vol. 41, no. 4, pp. 1-27, 2006.

[71] S. R. Cloude and K. P. Papathanassiou, "Polarimetric SAR interferometry," IEEE Trans. Geosci. Remote Sens., vol. 36, no. 5, pp. 1551-1565, Sep. 1998.

[72] K. P. Papathanassiou and S. R. Cloude, "Single-baseline polarimetric SAR interferometry," IEEE Trans. Geosci. Remote Sens., vol. 39, no. 11, pp. 2352-2363, Nov. 2001

[73] F. Ulaby, R. T. Moore, and A. K. Fung, Microwave Remote Sensing: Active and Passive. Norwood, MA: Artech House, 1986.

[74] G. Krieger, K. Papathanassiou, and S. Cloude, "Spaceborne polarimetric SAR interferometry: Performance analysis and mission concepts," EURASIP J. Appl. Signal Process., vol. 20, pp. 3272-3292, 2005

[75] A. Moccia, N. Chiacchio, and A. Capone, "Spaceborne bistatic synthetic aperture radar for remote sensing applications," Int. J. Remote Sens., vol. 21 , no. 18 , pp. 3395-3414, Dec. 2000. 
[76] G. Krieger and A. Moreira, "Spaceborne bi- and multistatic SAR: Potential and challenges," Proc. Inst. Electr. Eng.-Radar, Sonar Navig., vol. 153, no. 3, pp. 184-198, Jun. 2006.

[77] P. Dubois-Fernandez, H. Cantalloube, B. Vaizan, G. Krieger, R. Horn, M. Wendler, and V. Giroux, "ONERA-DLR bistatic SAR campaign: Planning, data acquisition, and first analysis of bistatic scattering behavior of natural and urban targets," Proc. Inst. Electr. Eng.—Radar, Sonar Navig., vol. 153 , no. 3, pp. 214-223, Jun. 2006.

[78] C. Prati and F. Rocca, "Improving slant-range resolution with multiple SAR surveys," IEEE Trans. Aerosp. Electron. Syst., vol. 29, no. 1, pp. 135-143, Jan. 1993.

[79] G. Krieger, N. Gebert, and A. Moreira, "Unambiguous SAR signal reconstruction from nonuniform displaced phase center sampling," IEEE Geosci. Remote Sens. Lett., vol. 1, no. 4, pp. 260-264, Oct. 2004.

[80] M. Suess, B. Grafmueller, and R. Zahn, "A novel high resolution, wide swath SAR system," in Proc. IGARSS, Sydney, Australia, 2001, pp. 1013-1015.

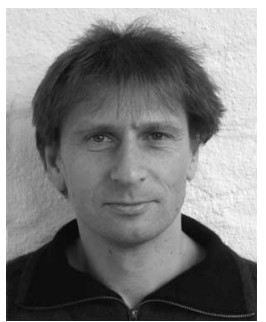

Gerhard Krieger (M'03) received the Dipl.-Ing. (M.S.) and the Dr.-Ing. (Ph.D.) degrees (honors) in electrical and communication engineering from the Technical University of Munich, Munich, Germany, in 1992 and 1999, respectively.

From 1992 to 1999 , he was with the LudwigMaximilians University, Munich, where he was an Interdisciplinary Research Scientist on the modeling of biological and technical vision systems. Since 1999, he has been with the Microwaves and Radar Institute (HR), German Aerospace Center (DLR), Oberpfaffenhofen, Germany. Since 2001, he has been the Head of the New SAR Missions Group at DLR (HR), and he is also currently appointed as the Systems Engineer of the TanDEM-X mission. His research interests include the development of innovative remote sensing system concepts based on radar interferometry, tomography, bi- and multistatic satellite formations, digital beamforming, as well as advanced signal and image processing techniques.

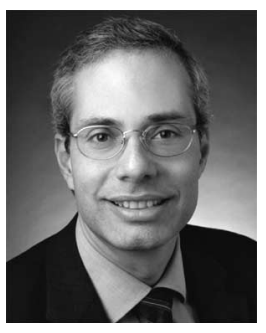

Alberto Moreira (M'92-SM'96-F'04) was born in São José dos Campos, Brazil, in 1962. He received the B.S. and M.S. degrees in electrical engineering from the Aeronautical Technological Institute, São José dos Campos, in 1984 and 1986, respectively, and the Eng.Dr. degree (honors) from the Technical University of Munich, Munich, Germany, in 1993

In 2003, he received a Full Professorship from the University of Karlsruhe, Germany, in the field of microwave remote sensing. As its Chief Scientist and Engineer, he managed the SAR Technology Department, Microwaves and Radar Institute, German Aerospace Center (DLR) from 1996 to 2001. Under his leadership, the DLR airborne SAR system, which is called E-SAR, has been upgraded to operate in innovative imaging modes like polarimetric SAR interferometry and SAR tomography. Since 2001, he has been the Director of the Microwaves and Radar Institute, DLR, Oberpfaffenhofen, Germany. The institute contributes to several scientific programs and space projects for actual and future air- and spaceborne SAR missions. Recently, the mission proposal TanDEM-X that is led by his institute has been approved for the realization phase. He is the Principal Investigator for this mission. His professional interests and research areas encompass radar end-to-end system design and analysis, innovative microwave techniques and system concepts, signal processing, and remote sensing applications.

Prof. Moreira is a member of the IEEE GRSS Administrative Committee (1999-2001 and 2004-2006), is the Chair of the German Chapter of the GRSS since 2003, and is actively serving as an Associate Editor for the IEEE Geoscience And Remote Sensing LetTers. Since 2003, he has been also a member of the Board of Directors of the Information Technology Society of German Association for Electrical, Electronic, and Information Technologies. He has been contributing to the successful series of the European SAR conferences since 1996 as a member of the Technical Program Committee, the Technical Chairman (2000), the Awards Chairman (2002-2004), and the General Chairman (2006). In 1995, he was the recipient of the DLR Science Award. He and his colleagues received the GRSS Transactions Prize Paper Awards in 1997 and 2001, respectively. He was also the recipient of the IEEE Nathanson Award (1999) and the IEEE Kiyo Tomiyasu Award (2007).

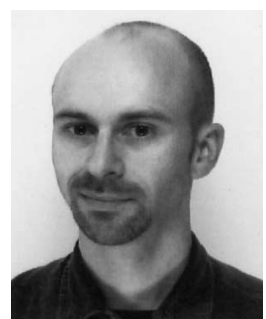

Hauke Fiedler received the Diploma degree in physics and the Dr. degree in astronomy from the Ludwig-Maximilians-Universität of Munich, Munich, Germany, in 1994 and 2000, respectively.

From 1994, he was working in the Cataclysmic Binary Group, Institute of Astronomy and Astrophysics with his work focused on hard and soft Xray interacting binaries, echo tomography, accretion disks, and related subjects. Since 2001, he has been with the Microwaves and Radar Institute, German Aerospace Center, Oberpfaffenhofen. He is working on future satellite missions for remote sensing with SAR, particularly on formation and configuration concepts, bi- and multistatic SAR performance analyses, and satellite mechanics.

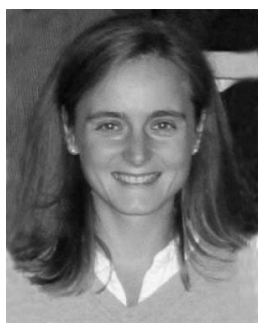

Irena Hajnsek (A'01-M'07) received the Dipl. degree (honors) from the Free University of Berlin, Berlin, Germany, in 1996, and the Dr. degree (honors) from the Friedrich Schiller University of Jena, Jena, Germany, in 2001.

From 1996 to 1999, she was a Project Scientist with the Microwaves and Radar Institute (DLR-HF), German Aerospace Center (DLR), Oberpfaffenhofen. From 1999 to 2000, she was an EU Fellow at the Institut d'Electronique et de Télécommunications de Rennes, University of Rennes I, France, and at the Applied Electromagnetics, St. Andrews, U.K. In 2000 , she was rejoined the DLR-HF, DLR. Since 2002, she has been leading the Polarimetric SAR Interferometry Research Group. In 2005, she was a Visiting Scientist at the University of Adelaide, Australia. She is responsible for the polarimetric science exploration of TerraSAR-X and Coordinator of the TanDEM-X Science Team. Her main research interests are in electromagnetic propagation and scattering theory, radar polarimetry, SAR and interferometric SAR data processing techniques, environmental parameter modeling, and estimation.

Dr. Hajnsek was the recipient of the DLR Science Award In 2002.

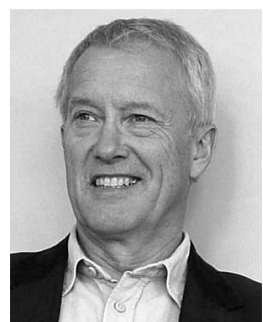

Marian Werner was born in Traunstein, Germany, in 1947. He received the Dipl.Ing. degree in communications engineering from the University of Applied Sciences in Munich, Munich, Germany, and the Dipl.Ing. degree in electrical engineering and microwave technology from the Technical University Berlin, Berlin, Germany.

Since 1975, he has been with the Microwaves and Radar Institute, German Aerospace Center (DLR), Oberpfaffenhofen, where he currently leads the Spaceborne SAR Systems Department. From 1975 to 1978 , he developed an L-Band scatterometer for airborne and ground experiments to measure the ocean wave spectrum. From 1978 to 1986, he was the Project Engineer for the European Microwave Remote Sensing Experiment which had a radiometer, scatterometer, and a synthetic aperture radar onboard the Space Shuttle STS 9, which is the first Space Laboratory flight. From 1986 to 1992, he was the Head of the Radar Remote Sensing Systems Group and in charge of the design specification for the Shuttle Radar Lab X-SAR system and the development of the DLR airborne SAR system E-SAR. From 1985 to 1991, he was a Technical Consultant with the German Space Agency. He supported the development and acceptance testing of the X-SAR space instrument. From 1991 to 1994, he was the X-SAR Project and Mission Operations Manager in the two SIR-C/X-SAR Space Shuttle missions (STS $59+68$ ). Since 1994, he has been the Leader and the Project Manager for the X-SAR/SRTM project with its successful mission in February 2000. During the preparation and the mission at NASA's Johnson Space Center, he acted as the X-SAR/SRTM Mission Manager (STS 99). Since March 2000, he has been also coordinating the $\mathrm{X}$-SAR/SRTM group of international scientists as the Project Scientist. Since 2002, he has been the Head of the Satellite SAR Systems Department which supports the TerraSAR-X radar satellite and future SAR system developments like TanDEM-X and other spaceborne SAR systems.

Mr. Werner received the AESS "Harry Rowe Mimno Award" for his SIR-C/ $X-S A R$ publication in the IEEE AEROSPACE AND EleCtronic SySTEM MAGAZINE in 1995 and the Wernher von Braun Ehrung for the SRTM Team from the Deutsche Gesellschaft für Luft- und Raumfahrt, Berlin, in April 2002. 


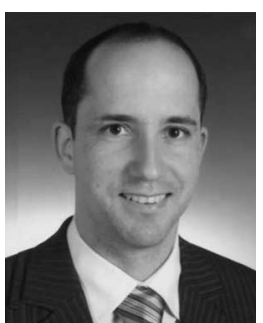

Marwan Younis (S'95-M'05) was born in Las Cruces, NM, in 1970. He received the B.Sc. degree in electronics and communication engineering from the University of Baghdad, Baghdad, Iraq, in 1992. He continued his studies in Germany, where he received the Diploma (Dipl.-Ing.) and the Dr.-Ing. (Ph.D.) degrees in electrical engineering from the University of Karlsruhe (TH), Karlsruhe, Germany, in 1997 and 2004, respectively.

From 1998 to 2004, he was a Research Scientist with the Institut für Höchstfrequenztechnik und Elektronik, TH. Since 2005, he has been with the Microwave and Radar Institute, German Aerospace Center, Oberpfaffenhofen. He is the author or coauthor of over 30 conference papers and ten reviewed publications. His research fields include SAR systems, digital beamforming for radar, bistatic SAR, and antennas.

Dr. Younis has served as Treasurer and Chair of the IEEE Student Branch in Karlsruhe. He is a member of the German Association for Location and Navigation. He is a Lecturer for Advanced Radio Communication and Spaceborne SAR Remote Sensing at TH. He received the Hermann-Billing Award for his Ph.D. thesis in 2005.

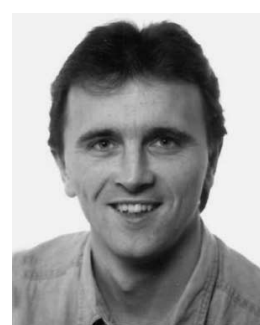

Manfred Zink received the Dipl.-Ing. degree in physics from the Technical University of Graz, Graz, Austria, in 1987, and the Dr.-Ing. degree from the University of Stuttgart, Stuttgart, Germany, in 1993.

In 1988, he was with the Microwave and Radar Institute, German Aerospace Center (DLR). He has pioneered the calibration techniques for both air- and spaceborne SAR sensors and was responsible for building up the Oberpfaffenhofen calibration site. He was the Lead X-SAR Calibration Engineer for both SIR-C/X-SAR missions in 1994 and for the SRTM mission in 2000. In August 2000, he was with the European Space Agency (ESA) and took over the responsibility for the calibration/validation of the ASAR onboard the ENVISAT satellite. After successful in-orbit commissioning of the ASAR, he was appointed as the Principal System Engineer for Phase B of ESA's TerraSAR-L Program. In May 2005, he returned to the Microwaves and Radar Institute, DLR, Oberpfaffenhofen, Germany, where he is currently heading the Microwave Systems Department. He is also managing the TanDEM-X Ground Segment development, which is a joint project performed by DLR's Center of Excellence for Advanced High-Resolution and 3-D SAR Technologies and Applications. 